\title{
Molecular characterization of the surface excess charge layer in droplets
}

\author{
Victor Kwan and Styliani Consta* \\ Department of Chemistry, The University of Western Ontario, London, Ontario, Canada \\ N6A $5 B^{r} 7$ \\ E-mail: sconstas@uwo.ca
}

\section{Abstract}

The surface excess charge layer (SECL) in droplets has often been associated with distinct chemistry. We examine the effect of the nature of ions in the composition and structure of SECL, by using molecular dynamics. We find that in the presence of simple ions the thickness of SECL is invariant not only with respect to droplet size but also with respect to the nature of the ions. In the presence of simple ions this layer has a thickness of $\approx 1.5-1.7 \mathrm{~nm}$ but in the presence of macroions it may extend to $\approx 2.0 \mathrm{~nm}$. The proportion of ions contained in SECL depends on the nature of the ions and the droplet size. For the same droplet size, $\mathrm{I}^{-}$and model $\mathrm{H}_{3} \mathrm{O}^{+}$ions show considerably higher concentration than $\mathrm{Na}^{+}$and $\mathrm{Cl}^{-}$ ions. We identify the maximum ion concentration region, which, in nanodrops, may partially overlap with SECL. As the relative shape fluctuations decrease when micro-drop size is approached, the overlap between SECL and maximum ion concentration region increases. We suggest the extension of the bi-layer droplet structure assumed in the equilibrium partitioning model of C. Enke to include the maximum ion concentration region that may not coincide with SECL in nanodrops. We compute the ion concentrations in SECL, which are those that should enter the kinetic equation in the ionevaporation mechanism, instead of the overall drop ion concentration that has been used.

\section{Introduction}

Native mass spectrometry often relies on the use of charged droplets to carry analytes from a sample into the gaseous phase. ${ }^{1-11}$ The charged droplets may originate from a liquid solution or from a solid matrix as in the recently developed matrix-assisted ionization (MAI) technique ${ }^{12,13}$ that combines features of matrix assisted laser desorption/ionization (MALDI) and electrospray ionization (ESI). These droplets comprise solvent, analytes, ions and possibly other additives. The initially sprayed droplets disintegrate as they are directed in their motion by an electric field through higher to lower pressure compartments. The purpose of the process is the release of analyte as free as possible from adducts and solvent molecules. In order to explain the mass spectra abundances intuitive macroscopic models of the droplet fragmentation kinetics, of its structure and of the effect of solvent evaporation have been developed over many decades. ${ }^{14-24}$ Despite the considerable amount of research that has been performed, the structure of charged droplets is still not completely analyzed and the mechanisms of charging of macroions are still under investigation.

In a series of articles we dissect the droplet structure using atomistic modeling with the aim to find the mechanisms of charging of macromolecules in spray-based methods used in native mass spectrometry and explain the acceleration of reactions in microdrops. ${ }^{25-30}$ In 
previous research we computed ion distribution profiles and compared them with our analytical theory that combines main factors that determine these distributions. ${ }^{31}$ These factors include a decay length of the ion distribution that arises from the solution of non-linear PoissonBoltzmann (NPB) equation for a rigid spherical droplet, shape fluctuations and the size of the ions. ${ }^{31}$ In the same article ${ }^{31}$ we developed methodology on how to compute the average and instantaneous electric field on the droplet surface using the multipole expansion method of electrostatics. ${ }^{31}$ Following these studies we addressed the question of the convergence of electrostatic properties in droplets. ${ }^{32}$ A systematic study of a variety of droplet sizes and use of scaling, allowed us to find universal behavior for the maximum of the ion distribution as a function of droplet size and extrapolate data to larger droplet sizes that cannot be studied by atomistic modeling thus far. ${ }^{32}$ By extrapolation, we found that the relative droplet shape fluctuations become negligible for droplet radius greater than $\approx 10 \mathrm{~nm}$.

In this article we extend our studies in addressing the droplet structure. Specifically, we study (a) the effect of the nature of ions and macroions in the composition and the width of surface excess charge layer (SECL); (b) the maximum ion concentration region (MICR) and (c) the molecular structure of SECL.

Below we present schematically the main results on droplet structure and the extension that we discuss in this article. We have found a bi-layer structure of an aqueous charged droplet by using atomistic modeling, ${ }^{32}$ as shown in the schematic in Fig. 1. Two regions are distinguished: an inner droplet region (bounded by the inner dotted circle) where the total charge arising from ions, hydrogen and oxygen sites of water molecules is zero and an outer region that carries the surface charge. Figure 1 also depicts a schematic representation of water density (blue line), radial distribution of simple ions with positive and negative charge (red lines) and the total charge distribution (solid black lines) that arises from the ions, hydrogen and oxygen sites of water. We have found ${ }^{32}$ that in the presence of $\mathrm{Na}^{+}$or $\mathrm{Cl}^{-}$ions, the to-

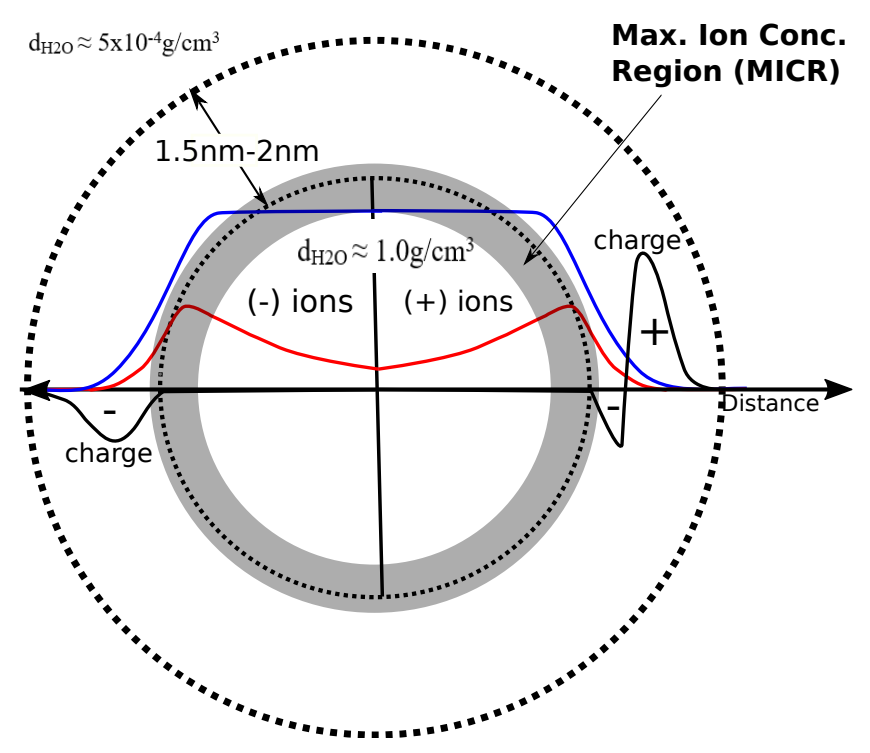

Figure 1: Schematic representation of a bi-layer structure of a droplet (represented by circles) as determined by atomistic simulations of aqueous droplets. ${ }^{32}$ The horizontal axis measures distance from a droplet's center of mass (COM). The perpendicular axis to the horizontal divides the schematic into two parts where the radial distribution of the positive ions is shown in the right semi-circle and that of the negative ions in the left semi-circle. The crossing of the axes is at the droplet's COM. The water mass density is shown by solid blue line, the ion radial distribution normalized by the volume of the spherical shell by red line and the total charge distribution (arising from H, O, ions) by solid black line. The inner dotted black line marks the onset of the building-up of the charge distribution and the outer dotted line is the droplet's boundary at a water density $\approx 5 \times 10^{-4} \mathrm{~g} / \mathrm{cm}^{3}$, where the charge density decreases to zero. The grey ring delimits the maximum ion concentration region (MICR) for which details are provided in the text.

tal droplet excess charge is located in an outer layer with thickness of $1.5 \mathrm{~nm}-1.7 \mathrm{~nm}$. Computations of various droplet sizes comprised $\mathrm{H}_{2} \mathrm{O}$ and $\mathrm{Na}^{+}$or $\mathrm{Cl}^{-}$ions showed that the percentage of ions in this layer depends on the droplet size. In droplets with diameter $<4 \mathrm{~nm}, \geq 55 \%$ of the total number of ions $\left(\mathrm{Na}^{+}\right.$or $\left.\mathrm{Cl}^{-}\right)$that corresponds to a concentration of $\geq 0.08 \pm 0.03 \mathrm{M}$, reside in SECL. In droplets with diameter > 
$16 \mathrm{~nm}$, the proportion is $\leq 24 \%$, which corresponds to a concentration of $\leq 0.03 \pm 0.01 \mathrm{M}$. The concentrations reported here have been estimated by dividing the moles of ions with the volume of the spherical shell where they reside. We note that SECL includes the droplet surface fluctuations, which leads to partial occupancy of the volume of the spherical shell with solvent. If we divide the moles of ions in SECL with the volume of the average number of $\mathrm{H}_{2} \mathrm{O}$ molecules in the spherical shell, then the concentration of the ions in the outer layers is higher, but still the new concentration is not dramatically higher than those estimated by using the entire spherical shell.

In this article we show that the percentage of ions in SECL depends on its nature. In the presence of macromolecules it is possible that SECL has a thickness of $2.0 \mathrm{~nm}$. For this reason in Fig. 1 we indicate thickness $1.5 \mathrm{~nm}-2.0 \mathrm{~nm}$ instead of $1.5 \mathrm{~nm}-1.7 \mathrm{~nm}$ that we found for simple ions in previous works. ${ }^{32}$ Depending on the nature of the ion SECL may not hold the highest concentration of ions. We identify the maximum ion concentration region (MICR), which partially overlaps with SECL. The combination of these two regions may be responsible for distinct chemistry in droplets because this broader region is characterized by higher ion concentration than a bulk analogue, surface fluctuations that may couple with the reaction mechanisms, ${ }^{33}$ existence of free surface that may facilitate the reaction ${ }^{33}$ and distinct molecular structure, which is more pronounced as the droplet becomes smaller.

Our computational bi-layer droplet structure is in agreement with the assumptions of the equilibrium partitioning model (EPM) of C. Enke. ${ }^{22,34}$ EPM assumes two regions in a droplet, a core region, and an outer region that has the surface charge. Since EPM is a macroscopic model, it cannot readily provide the thickness of the outer layer and the amount of free charge present, thus our molecular modeling provides complementary information in that respect. Enke points out that the surface excess charge layer is a "different phase" in the droplet where distinct chemistry takes place. Here, we propose that within the frame- work of EPM one may consider that the important region for chemical reactivity is extended beyond SECL to include the region where the concentration of the ions is still significant.

We remind that another factor that may affect the analyte concentration on the droplet surface is the solvent evaporation rate. A detailed discussion of the effect of the evaporation rate is found in our previous articles. ${ }^{31}$ In this situation, the competition between solvent evaporation rate and analyte diffusion rate will determine whether the analyte concentration will increase on the surface because of kinetic trapping. In order to study these effects by direct molecular dynamics evaporation of droplets of radius $>10 \mathrm{~nm}$ should be performed so as there is enough time for the history of the droplet to play a role in the manner that an analyte is released from a droplet. To our knowledge these studies are not feasible yet. However, if evaporative cooling leads to droplets at room temperature then at this droplet size the evaporation rate is slow enough to justify the use of equilibrium simulations to unravel their structure.

In this article we consider droplets that contain a number of separate charges. For simulating the droplet equilibrium structure, that requires a constant droplet size, the solvent evaporation is suppressed by including the droplet in a volume where it is in equilibrium with its vapor. When the ratio of the droplet charge squared to its volume is below a threshold value the droplet is in a metastable state. This threshold value is called the Rayleigh limit. The condition for non-spontaneous vs spontaneous droplet break-up is given by the Rayleigh fissility parameter $(X)$ defined as

$$
X=\frac{Q^{2}}{64 \pi^{2} \gamma \varepsilon_{0} R^{3}}
$$

where $Q$ is the droplet charge, $\gamma$ the surface tension, $\varepsilon_{0}$ and $R$ are the permittivity of vacuum and the radius of the droplet, respectively. When $X=1$ the system is at the Rayleigh limit. ${ }^{35-39}$ The Rayleigh limit yields an estimate of the largest amount of charge a droplet of certain volume can sustain without fragmenting 
spontaneously. ${ }^{40}$ The Rayleigh limit is used in this article to justify the amount of charge in the droplets that we simulate. The droplet radius at the Rayleigh limit will be denoted as $R_{\mathrm{r}}$ and the corresponding charge $Q_{\mathrm{r}}$. Therefore, $X$ can be also expresses as

$$
X \approx\left(\frac{Q}{Q_{\mathrm{r}}}\right)^{2} .
$$

\section{Models and Simulation Methods}

We performed equilibrium molecular dynamics (MD) simulations of charged aqueous droplets with simple ions which include $\mathrm{Na}^{+}, \mathrm{Cl}^{-}, \mathrm{I}^{-}$, model hydronium ions and with a macroion using the example of a cholera toxin ${ }^{41}$ pentamer in the charge state of The simulations were performed by using the software NAMD version 2.12. ${ }^{42}$ Newton's equation of motion for each atomic site was integrated using the velocity-Verlet algorithm with a time step of 1.0 fs. The trajectories were analyzed using VMD 1.9.2. ${ }^{43}$ The water molecules were modeled with the TIP3P (transferable intermolecular potential with 3 points) ${ }^{44}$-CHARMM and the TIP $4 \mathrm{P} / 2005^{45}$ and the simple ions with the CHARMM36m ${ }^{46,47}$ and OPLS (Optimized Potentials for Liquid Simulations), ${ }^{48,49}$ respectively. The TIP3P-CHARMM is a $\mathrm{m}$ (odified)TIP3P, which is the original TIP3P with Lennard-Jones potential on the hydrogen sites. Hereafter we will use the notation TIP3P for this water model. Details of the molecular parameters and the models used for each system are presented in Table S1 and Table S2, respectively, in SI. Table 1 shows the systems that are simulated and physical parameters that characterize them.

The large number of studies on the Eigen ${ }^{50}$ and Zundel ${ }^{51}$ complexes of water with hydronium ion in small clusters are indicative of the challenges in modeling and experiments. ${ }^{52-72}$ Because of the large droplets that we study, we model the hydronium ions by an empirical model, where the parameters were taken from. ${ }^{73}$ The testing of the model is presented in Fig. S1 in SI. Aqueous droplets with $\approx 880$ TIP3P $\mathrm{H}_{2} \mathrm{O}$ molecules and 6 hydronium ions at $T=300 \mathrm{~K}$ and $T=350 \mathrm{~K}$ were studied. We address the following questions: (i) Is there enough space on the droplet surface to accommodate the Eigen "pyramids". (ii) Does the particular structure of the Eigen complex, differentiate its location in a droplet relative to that of $\mathrm{Na}^{+}$ions?

We performed molecular dynamics simulation of a cholera toxin pentamer with the RCSB $\mathrm{PDB}^{74,75}$ ID 1 JR0 in the charge state of $+25 e$ in an aqueous droplet comprised $\sim 2 \times 10^{4}$ TIP $3 \mathrm{P}$ water molecules and $14 \mathrm{Na}^{+}$ions at $T=350 \mathrm{~K}$. The protomers in the pentameric structure are identical and the protein assembly overall has toroidal. We studied three systems (Table S2 in SI): i) with no counterions, ii) with 18 pairs of $\mathrm{Na}^{+}$and $\mathrm{Cl}^{-}$, which correspond to a counterion concentration of $50 \mathrm{mM}$ and iii) with 36 pairs of $\mathrm{Na}^{+}$and $\mathrm{Cl}^{-}$, which correspond to a counterion concentration of $100 \mathrm{mM}$. The radius of the droplet is $5.6 \mathrm{~nm}$ and the total charge of the system is $+40 e$.

Equilibrium simulations in NAMD were set by placing the droplet in a spherical cavity of radius $20.0 \mathrm{~nm}$ by using spherical boundary condition. The cavity was sufficiently large to accommodate the shape fluctuation of the droplet. The droplet will eventually reach vapor pressure equilibrium. The systems were thermalized with Langevin thermostat with the damping coefficient set to $1 /$ ps. All the forces were computed directly without any cut-offs. The Rayleigh limit of the droplet (Eq. 1 for $X=1$ ) was calculated with the simulation determined surface tension values of the specific water model at the simulation temperature. ${ }^{76}$ Specifically, for TIP3P at $T=300 \mathrm{~K}$ the value of surface tension is taken to be $0.0523 \mathrm{~N} / \mathrm{m}$ and at $T=350 \mathrm{~K}$ to be $0.0432 \mathrm{~N} / \mathrm{m}$. For TIP4P $/ 2005$ at $T=350 \mathrm{~K}$ surface tension is taken to be $0.0619 \mathrm{~N} / \mathrm{m}$. We point out that we only use the surface tension values to compute the fissility parameter (Eq. 1), and thus provide an estimate of the degree of droplet stability.

The dimension of a droplet (with or without ions) is often measured by the equimolecular radius $\left(R_{\mathrm{e}}\right)$ (values for various systems are found 
Table 1: Systems studied, characteristic dimensions and concentrations. $n_{\mathrm{H}_{2} \mathrm{O}}$ denotes average number of water molecules and $n_{\mathrm{I}}$ number of ions in the droplets during the production runs. $R_{\mathrm{e}}[\mathrm{nm}]$ is the equimolecular radius, ${ }^{32}$ computed by using density of the TIP3P model at $350 \mathrm{~K}$ to be $0.9539 \mathrm{~g} / \mathrm{cm}^{3} \cdot r_{\max }[\mathrm{nm}]$ is the distance from the droplet center of mass (COM) to the maximum of the ion concentration profile. $\lambda_{\mathrm{PB}}$ $[\mathrm{nm}]$ is a characteristic decay length of the ion concentration that emerges from the solution of the non-linear Poisson-Boltzmann equation. Details for $\lambda_{\mathrm{PB}}$ are presented in the text. $C_{\mathrm{MICR}}[\mathrm{M}]$ (where $\mathrm{M}$ stands for molarity) is the concentration of the ions in the maximum ion concentration region (details are presented in the text) and $C_{\mathrm{SECL}}$ $[\mathrm{M}]$ is the concentration in SECL. $C_{\mathrm{b}}[\mathrm{M}]$ is the bulk ion concentration estimated by dividing the number of moles of ions by the volume of a droplet of radius $R_{\mathrm{e}}$. $X$ is the fissility parameter of the simulated droplet (Eq. 1). "_" means that the quantity has not been defined for a certain droplet size (details are presented in the text). In all the systems the temperature is set at $T=350 \mathrm{~K}$.

\begin{tabular}{|c|c|c|c|c|c|c|c|}
\hline$n_{\mathrm{H}_{2} \mathrm{O}}$ & $n_{\mathrm{I}}$ & $R_{\mathrm{e}}[\mathrm{nm}]$ & $r_{\max }[\mathrm{nm}]$ & $\lambda_{\mathrm{PB}}[\mathrm{nm}]$ & $C_{\mathrm{MICR}}[\mathrm{M}]$ & $C_{\mathrm{SECL}}[\mathrm{M}]$ & $C_{\mathrm{b}}[\mathrm{M}]$ \\
\hline 880 & $1 \mathrm{Na}^{+}$ & 1.88 & $0.00-0.80$ & - & - & $0.010 \pm 0.001$ & 0.006 \\
880 & $1 \mathrm{Cl}^{-}$ & 1.88 & $0.00-0.80$ & - & - & $0.010 \pm 0.001$ & 0.006 \\
880 & $6 \mathrm{Cl}^{-}$ & 1.88 & $1.00-1.05$ & 0.76 & & - & 0.36 \\
3500 & $16 \mathrm{Cl}^{-}$ & 2.95 & $2.20-2.25$ & 0.70 & $0.33 \pm 0.1$ & $0.058 \pm 0.006$ & 0.247 \\
$3 \times 10^{4}$ & $44 \mathrm{Cl}^{-}$ & 6.08 & $5.05-5.10$ & 1.08 & $0.103 \pm 0.003$ & $0.029 \pm 0.003$ & 0.078 \\
3500 & $15 \mathrm{I}^{-}$ & 2.95 & $2.45-2.50$ & 0.75 & $0.276 \pm 0.008$ & $0.075 \pm 0.008$ & 0.231 \\
\hline 880 & $6 \mathrm{H}_{3} \mathrm{O}^{+}$ & 1.875 & $1.60-1.65$ & 0.76 & - & $0.112 \pm 0.001$ & 0.360 \\
\hline $2 \times 10^{4}$ & $1 \mathrm{JR}^{25+}$ & 5.5 & & - & & & \\
& $14 \mathrm{Na}^{+}$ & & $4.50-4.55$ & - & - & $0.010 \pm 0.001$ & 0.038 \\
$2 \times 10^{4}$ & $1 \mathrm{JR}^{25+}$ & 5.5 & & - & & & \\
& $32 \mathrm{Na}^{+}$ & & $4.40-4.45$ & - & $0.103 \pm 0.003$ & $0.029 \pm 0.003$ & 0.089 \\
& $18 \mathrm{Cl}^{-}$ & & $3.70-3.75$ & - & $0.0713 \pm 0.002$ & $0.018 \pm 0.002$ & 0.049 \\
$2 \times 10^{4}$ & $1 \mathrm{JR0}^{25+}$ & 5.5 & & - & & & \\
& $50 \mathrm{Na}^{+}$ & & $4.60-4.65$ & - & $0.166 \pm 0.004$ & $0.047 \pm 0.005$ & 0.138 \\
& $36 \mathrm{Cl}^{-}$ & & $3.25-3.30$ & - & $0.143 \pm 0.004$ & $0.014 \pm 0.002$ & 0.100 \\
\hline
\end{tabular}


in Table 1). This radius is determined from the zero adsorption condition. ${ }^{77}$ If the vapor density is approximated to zero, $R_{\mathrm{e}}$ can be given as:

$$
R_{\mathrm{e}}=\left(\frac{3 N}{4 \pi \rho^{l}}\right)^{1 / 3}
$$

where $N$ is the number of molecules in the droplet, $\rho^{l}$ is the density of the bulk liquid.

\section{Use of microcanonical or canoni- cal ensemble in the droplet simu- lations?}

In comparing experimental with simulation data one should consider the following questions. (a) What is the temperature in experiments of a nano-drop of the size that can be atomistically simulated? (b) What ensemble should be used in equilibrium simulations, the canonical or the microcanonical ensemble? The temperature of the droplets in ESI experiments depends on the experimental conditions and the instrument used. Insightful experiments performed by Antoine et al. ${ }^{78}$ and Cook et al. ${ }^{79}$ detect the temperature of micro-drops. Both studies agree that nanoscopic droplets will be found at elevated temperature because they will undergo faster thermalization with the warm background gas than the micro-drops. The relation of temperature between the simulated and experimental drops has been addressed by Consta et al. ${ }^{80}$ Even if experimental conditions are not exactly known, simulations can still generate the possible experimental scenarios. Our previous works showed that the radial ion distributions $^{31,32}$ are practically the same in the temperature range of $300 \mathrm{~K}$ to $350 \mathrm{~K}$ and we currently study the effect of very low temperature.

Now, we compare the microcanonical with the canonical ensemble simulations. The use of the two ensembles in isolated clusters comprised up to a few tens of molecules has been hotly debated over a decade. The consensus has been that the canonical and microcanonical ensemble result in the same structure of a cluster. Differences between the two ensembles appear in the caloric curves of small clusters and for this reason both ensembles are used in their computation. ${ }^{81,82}$ Several works have brought up issues with ergodicity and lack of Maxwellian distribution of atomic velocities in small clusters simulated in the microcanonical ensemble. ${ }^{83,84}$ Below we compare the simulation set-up used in this article of a droplet in equilibrium with its vapour within a cavity with that of an isolated droplet in vacuo. The former set-up allows us to assign a statistical mechanics ensemble (canonical or microcanonical) to the droplet-vapor system. Figure S2 in SI compares the total energy (sum of potential and kinetic energy), temperature and radial ion distributions using a microcanonical and canonical ensemble using a GPU processor. In the microcanonical simulations the total energy shows a drift relative to its constant value along the trajectory. The origin of the energy drift has been analyzed in many classic textbooks (see for example ${ }^{85}$ ) of molecular simulations. We find that the ion distribution is the same in the two ensembles. The energy drift is negligible in the CPU processor run of microcanonical MD because of the double precision used (Fig. S3 in SI). A microcanonical MD run may suffer from the known issues of energy drift that is affected by the integration algorithm and the numerical precision, and heating-up of the system even for a 2 fs time-step. The same issues appear in a microcanonical simulation of an isolated droplet (Fig. S4 in SI) but there the situation is even more problematic for reasons that are mentioned below.

From an experimental view, it might be considered that an isolated droplet (not at equilibrium with its vapor) simulated in the microcanonical ensemble is more representative of reality. The simulations of an isolated droplet have several known pitfalls. Firstly, it is not clear how to assign an ensemble to an isolated droplet because the droplet constantly evaporates. Secondly, in the course of a long trajectory as the one required to obtain converging ion distributions, the droplet quickly develops angular and linear momentum because of evaporation. As a result, the internal temperature of the droplet decreases significantly, leading to freezing. Angular and linear momenta are natural motions that are expected to appear in 
reality (of course, the angular and linear momentum will be affected by the collisions with background gas if it is present). We have found significant droplet evaporation even at as low a temperature as $280 \mathrm{~K}$ over a trajectory of several nanoseconds. One may delay the effect of the evaporation by a few nanoseconds if the droplet is very cold, as for example, an aqueous droplet of $880 \mathrm{H}_{2} \mathrm{O}$ molecules at $T=250 \mathrm{~K}$. Then a significant energy drift and heating-up because of numerical noise appear in $\approx 5 \mathrm{~ns}$ as shown in Fig. S4 in SI vs a few hudrends of picoseconds in similar droplets at room temperature. Microcanonical molecular dynamics in a time interval of nanoseconds is applied best at very low temperature where the effect of solvent evaporation is suppressed and in large droplets.

In conclusion, microcanonical and canonical ensemble provide the same ion distributions in simulations of droplets in equilibrium with vapor within a cavity. The microcanonical dynamics shows the already known issues of energy drift and system heating-up, which prevents from using a larger time step of 2 fs. Microcanonical dynamics for very long trajectories is not possible without the use of corrective methods (e.g. velocity rescaling), which of course, deviate the microcanonical ensemble from its ideal form. In isolated drops the solvent evaporation presents additional challenges in the use of the microcanonical ensemble because of the development of linear and angular momentum. Because of certain weaknesses of microcanonical MD in running long trajectories for achieving convergence of equilibrium properties, the canonical ensemble in a dropletvapor equilibrium system is preferred. Constant energy MD should be used in the study of fragmentation dynamics of charged droplets, but the microcanonical MD has to be used in combination with free energy surfaces along collective reaction coordinates as it has already been implemented in the release of single ions from droplets by Consta et al. ${ }^{86}$ in the study of the IEM mechanism. In this case, the MD trajectories are short (less than a few tens of picoseconds), because they are initiated at the barrier top (transition state) of the free energy profiles. Within the time of these short MD trajectories there is no energy drift, no development of angular or linear momentum and even if there is solvent evaporation it is still very minimal.

\section{Results and Discussion}

\section{Charge distribution in droplets with a single ion}

In order to obtain insight into the solvent structure in charged droplets, first we will examine an aqueous droplet with $880 \mathrm{H}_{2} \mathrm{O}$ molecules and a single $\mathrm{Na}^{+}$(droplet diameter $\approx 4 \mathrm{~nm}$ ). The solvation of a single ion has been extensively studied in the context of the Born model. ${ }^{87-95}$

Figure 2 (a) shows the radial ion distrtibution of a droplet comprised $\approx 880 \mathrm{H}_{2} \mathrm{O}$ molecules and a single $\mathrm{Na}^{+}$or single $\mathrm{Cl}^{-}$. The error bars for $\mathrm{Na}^{+}$in (a) is not shown for clarity, they are of the same magnitude as for $\mathrm{Cl}^{-}$. The profiles in Fig. 2 (a) have been normalized by dividing the raw histogram data with the volume of a spherical shell $\left(\frac{4}{3} \pi\left[(r+d r)^{3}-r^{3}\right]\right.$ where $r$ is the distance from the droplet COM).

Figure 2 (b) and (c) depict the nonnormalized charged distribution of a pristine aqueous droplet and that in the presence of a single $\mathrm{Na}^{+}$ion and a single $\mathrm{Cl}^{-}$ion, respectively. Figure 2 (b) shows that for a pure $\approx 880$ $\mathrm{H}_{2} \mathrm{O}$ droplet the charge distribution in the interval $[14.0 \pm 1 \AA, 18.0 \pm 1 \AA]$ (negative trough) integrates to $\approx-3.25 e$ and in $[19.0 \pm 1 \AA$, $25.0 \pm 1 \AA]$ (positive peak) to $\approx+3.21 e$. The total charge over the undulation is $\approx 0.0 e$ as expected. The same figure shows that in the presence of a $\mathrm{Na}^{+}$ion, solvent polarization appears in the droplet interior. This is not surprising since the $\mathrm{Na}^{+}$ion has a significant probability to be in the droplet interior. The integral of the polarization charge from the droplet $\mathrm{COM}$ to $17.0 \pm 1 \AA$ is $\approx-3.76 e$ and under the positive peak yields $\approx+3.75 e$. Thus, differently from the pure $\mathrm{H}_{2} \mathrm{O}$, in the presence of a single $\mathrm{Na}^{+}$ion the negative polarization charge is spread over a much broader interval (because of the ion diffusion) and it is $\approx 0.5 e$ lower from that of pure $\mathrm{H}_{2} \mathrm{O}$. The total charge distribution (arising from $\mathrm{H}, \mathrm{O}, \mathrm{Na}^{+}$) is also shown 


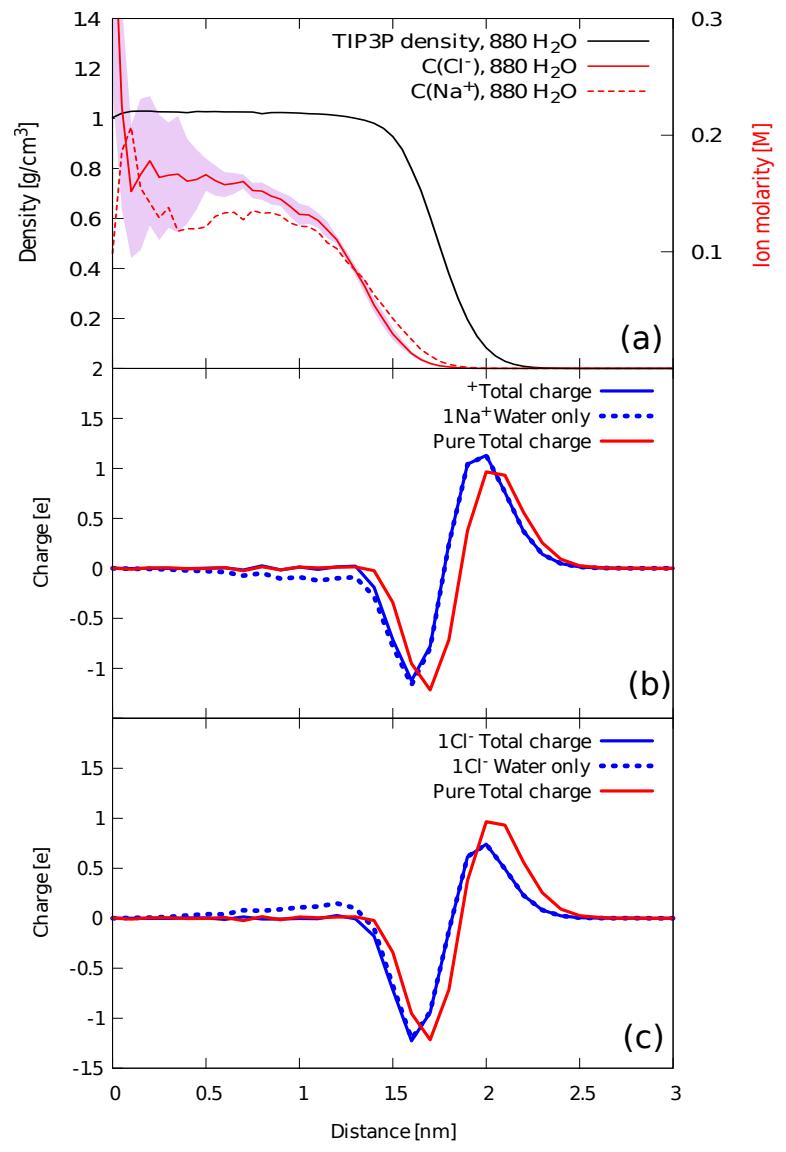

Figure 2: (a) Single $\mathrm{Na}^{+}$and $\mathrm{Cl}^{-}$radial distribution profiles (normalized) in a droplet comprised $\approx 880 \mathrm{H}_{2} \mathrm{O}$ molecules. Normalization is discussed in the text. The density of water is shown by the solid black line. (b) Charge radial distributions (normalization has not been applied) of the same size droplet as in (a) of a pristine droplet shown by the red solid line, of the water only (polarization charge) in the droplet with a single $\mathrm{Na}^{+}$ion by the blue dotted line and the total $\left(\mathrm{H}, \mathrm{O}, \mathrm{Na}^{+}\right)$by the blue solid line. (c) same as (b) but for $\mathrm{Cl}^{-}$. The bin size is $1.0 \AA$. Error bars are not shown in (b) and (c) for clarity. The plots with error bars are shown in Fig. S5 in SI. The origin of all the profiles is at the droplet COM.

in Fig. 2 (b). The integral over $14.0 \pm 1 \AA$ $17.0 \pm 1 \AA$ (negative trough) yields $\approx-2.8 e$ and in $18.0 \pm 1 \AA-25.0 \pm 1 \AA, \approx 3.76 e$. These differ- ences show that the $\mathrm{Na}^{+}$ion partially compensates the negative trough of the pure water, which integrates to $\approx-3.25 e$ vs $\approx-2.8 e$ in the presence of the ion. When the ion is in the droplet interior, the ion charge is compensated as indicated by the total charge of zero in the interior. The integral of the charge distribution (comprised $\mathrm{O}, \mathrm{H}$ sites) with center the single ion compensates the positive ion when integrated up to $8 \AA$. In the outer droplet layers the ion appears with higher probability (not normalized) at $\approx 13 \AA$ which is the distance at which the charge undulation starts to build up. The charge compensation will take place up to $20 \AA-21 \AA$. This distance corresponds to the top of the positive peak in Fig. 2 (b). Beyond this peak the density of water molecules is negligible, and the few water molecules form islands on top of the interior solvation shells. Figure 2 (c) is the same as Fig. 2 (c) but for a $\mathrm{Cl}^{-}$ion. A distinct feature is that the positive peak in the charge distribution of pristine water is reduced in the presence of the $\mathrm{Cl}^{-}$ion.

\section{Effect of the nature of the ion on the surface excess charge layer - Maximum ion concentration re- gion}

Figure 3 (a) shows the ion radial distribution of $\mathrm{Cl}^{-}$ions in droplets of $880 \mathrm{H}_{2} \mathrm{O}$ molecules- $6 \mathrm{Cl}^{-}$ $(X=0.57)$ and $3 \times 10^{4} \mathrm{H}_{2} \mathrm{O}$ molecules- $44 \mathrm{Cl}^{-}$ $(X=0.91)$ using the TIP3P model. The distribution of $\mathrm{Na}^{+}$ions has been reported in Ref. ${ }^{32}$ The region where the surface excess charge is found has been highlighted. The outer boundary of the highlighting stripe is found where the density of water is $\approx 5 \times 10^{-4} \mathrm{~g} / \mathrm{cm}^{3}$ and the inner boundary is where the surface excess charge starts to build-up (see also Fig. 1). The highlighted region contains 55\% (concentration $0.082 \mathrm{~mol} / \mathrm{L}$ ) and $34 \%$ (concentration $0.034 \mathrm{~mol} / \mathrm{L}$ ) of total number of $\mathrm{Cl}^{-}$ions in droplet of 880 and $3 \times 10^{4} \mathrm{H}_{2} \mathrm{O}$ molecules, respectively. We remind that the ion concentrations have been estimated by dividing the moles of ions with the volume of the spherical shell where they reside. SECL (highlighted region 


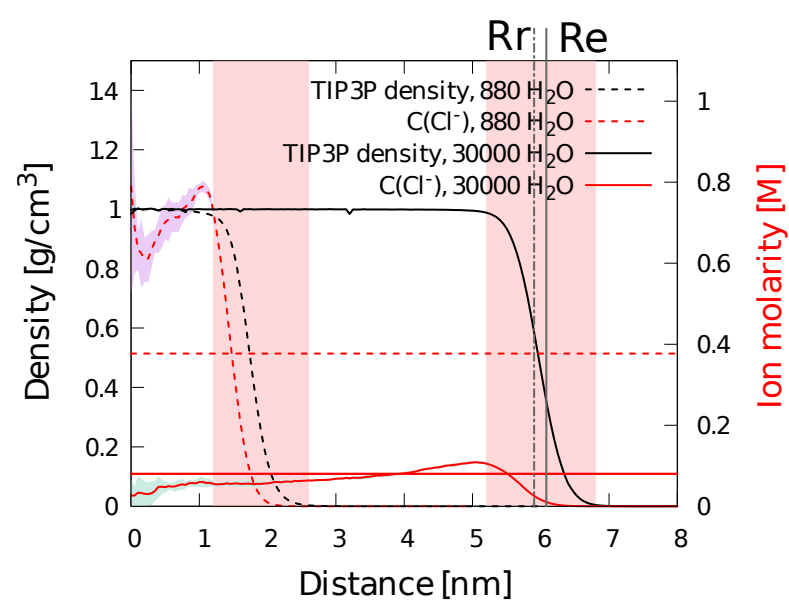

(a)

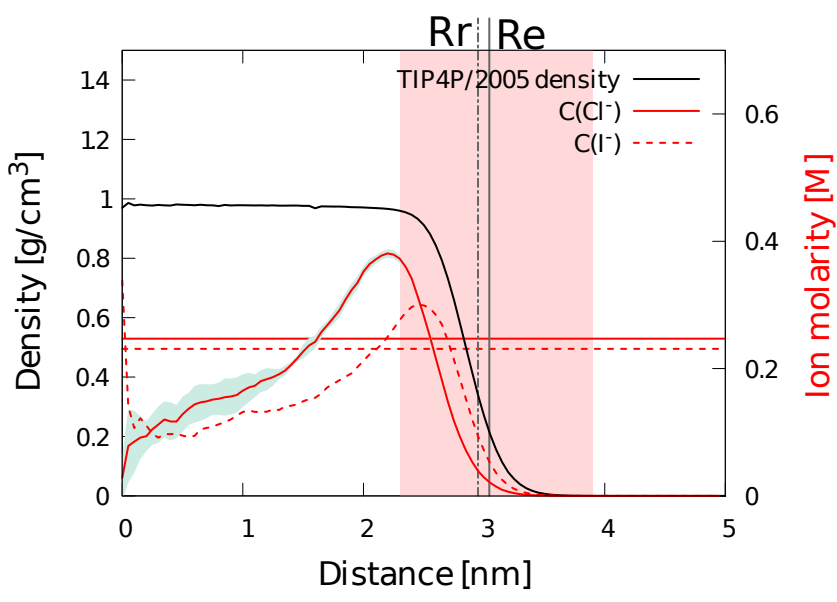

(b)

Figure 3: (a) $\mathrm{Cl}^{-}$concentration profiles and $\mathrm{H}_{2} \mathrm{O}$ density vs distance from the droplet COM for droplets of 880 and $3 \times 10^{4} \mathrm{H}_{2} \mathrm{O}$ molecules at $T=350 \mathrm{~K}$. TIP3P model is used. (b) Same as (a) but for $16 \mathrm{Cl}^{-}$and $16 \mathrm{I}^{-}$in a droplet of $3500 \mathrm{H}_{2} \mathrm{O}$ molecules. TIP4P/2005 for water and OPLS parameters for the ions are used. The horizontal lines mark the bulk ion concentration $C_{\mathrm{b}}$ (values are presented in Table 1 ). The vertical grey lines mark the Rayleigh radius, $R_{\mathrm{r}}$, for the amount of charge in the droplet and the equimolecular radius, $R_{\mathrm{e}}$ (values are presented in Table 1).

in Fig. 3 (a)-(b)) is strongly affected by shape fluctuations, thus this outer region is not filled with solvent. We can also estimate the concentration of the ions by using the average volume of the $\mathrm{H}_{2} \mathrm{O}$ molecules in the highlighted region.
Then the ion concentration is estimated to be higher in SECL, but still, it is not a dramatic change relative to the concentration profiles in Fig. 3 (a)-(b).

Figure 3 (b) is similar to (a) but for droplets comprised $3500 \mathrm{H}_{2} \mathrm{O}$ molecules and either 16 $\mathrm{Cl}^{-}$ions or $16 \mathrm{I}^{-}$ions. It is reminded that these systems have been modeled by TIP4P/2005 model for $\mathrm{H}_{2} \mathrm{O}$ and OPLS parameters for the ions (Table S2 in SI). SECL with thickness in the range of $24 \pm 1 \AA-38 \AA$ contains $36 \%$ of $\mathrm{Cl}^{-}$ions which corresponds to a concentration of $0.056 \mathrm{~mol} / \mathrm{L}$ and $54 \%$ of $\mathrm{I}^{-}$ions, which corresponds to $0.079 \mathrm{~mol} / \mathrm{L}$. The charge distribution of the systems with the $16 \mathrm{Cl}^{-}$ions or $16 \mathrm{I}^{-}$ions is shown in Fig. S6 in SI. Three interesting points arise from the study of these ion distributions. Firstly, the nature of the ion plays a significant role in the percentage of free ions in SECL $\left(36 \%\right.$ of $\mathrm{Cl}^{-}$ions vs. $54 \%$ of $\mathrm{I}^{-}$ ions). Secondly, the different percentage of ions in SECL and the nature of the ions does not affect its thickness. Thirdly, the SECL thickness using TIP4P/2005 model is found to be $1.6 \mathrm{~nm}$. In previous research ${ }^{32}$ we have found it in the range of $1.5 \mathrm{~nm}-1.7 \mathrm{~nm}$ using the TIP3P water model. We conclude that the thickness of SECL shows low sensitivity to the force field parameters.

At this point we discuss an intriguing observation that we made that allows us to identify the maximum ion concentration region (MICR) of a charged droplet and its relation to SECL. In previous articles we introduced the decay length of the ion distribution that arises from solving the non-linear Poisson-Boltzmann equation (NPB) for a rigid spherical droplet. ${ }^{31,32,96}$ This length, which we denote it as $\lambda_{\mathrm{PB}}$, looks in its form similar to the "Debye" length for neutral electrolytes. $\lambda_{\mathrm{PB}}$ for monovalent ions is given by

$$
\lambda_{\mathrm{PB}} \approx \frac{\epsilon k_{B} T}{\sigma}
$$

where $k_{B}$ is Boltzmann constant, $T$ is temperature, $T=350 \mathrm{~K}$ for the droplets we study, $\epsilon$ is the permittivity of water estimated using dielectric constant 61.7 for $T=350 \mathrm{~K}$ and $\sigma=\frac{|Z| e^{2}}{4 \pi R_{\mathrm{e}}^{2}}$ for ions of charge $\pm 1 e$, where $e$ is the charge of the electron and $|Z|$ is the abso- 
lute value of droplet charge in units of $e$. The values of $\lambda_{\mathrm{PB}}$ for the droplets we study are presented in Table 1. The NPB finds that the ion distribution has its maximum value on the spherical surface and decays exponentially toward the droplet interior. The NPB considers a rigid sphere, thus it ignores the effect of shape fluctuations that appear in the outer layers of a droplet. If we consider the difference $r_{\max }-\lambda_{\mathrm{PB}}$ (shown in Table 1) we find a characteristic distance from the droplet COM, where the ion distribution has decayed from its peak value. Let us make now the following construction: we define the ion concentration, which we call bulk concentration, $C_{\mathrm{b}}[\mathrm{M}]$ (shown in Table 1 ), of the droplet by simply dividing the moles of ions by the volume of a sphere with radius $R_{\mathrm{e}}$. The $C_{\mathrm{b}}$ concentration is shown by the horizontal line in Figs 3 (a)-(b). The intersection of the $C_{\mathrm{b}}$ line with the radial ion distribution towards the droplet interior coincides with the point with abscissa $r_{\max }-\lambda_{\mathrm{PB}}$. We have made this observation consistently not only for the ion distributions presented in Fig. 3 (a)-(b), but also for the data presented in Ref. ${ }^{32}$ However, this is the first time we report our observation. Table S3 in SI shows the values of $\lambda_{\mathrm{PB}}$ for systems we studied in Ref. ${ }^{32}$ This observation shows that $\lambda_{\mathrm{PB}}$, even though approximate, it defines a specific length of ion decay that has a solid theoretical foundation. The radial ion distribution at distance $>r_{\max }$ is strongly affected by shape fluctuations. The $C_{\mathrm{b}}$ line intersects this ion radial distribution in an outer point that is found within SECL. If we consider the portion of the ion distribution located between the two intersection points of the $C_{\mathrm{b}}$ line with the radial ion distribution, we define an important region within a droplet, which we call the maximum ion concentration region (MICR). The outer part of MICR overlaps with SECL. We note here two important points: (a) For droplets of $\approx 10^{3} \mathrm{H}_{2} \mathrm{O}$ molecules, $\lambda_{\mathrm{PB}} \approx r_{\max }$. This is consistent with our findings that the $\mathrm{x}$-scaled radial ion distribution is almost uniform for droplets of this size. ${ }^{32}$ For droplets of this size we cannot distinguish MICR concentration as indicated in Table 1. (b) $\lambda_{\mathrm{PB}}$ is defined via the NPB equation for a rigid droplet. In reality a droplet un- dergoes shape fluctuations. The larger or the colder a droplet the smaller the shape fluctuations relative to the spherical shape ${ }^{32}$ (also demonstrated in Fig. S7 in SI). Under these conditions MICR and SECL will show significant overlap. ${ }^{96}$

In summary, we identify two significant regions in the charged droplets: (a) SECL (surface excess charge layer) is strongly affected by shape fluctuations. We expect that this is the layer from which ions are released in IEM. In previous articles we have reported that shape fluctuations become negligible relative to the spherical shape at droplet radius greater than $10 \mathrm{~nm} .{ }^{32}$ This droplet size coincides with that below which IEM is expected to dominate Rayleigh fission. Our results indicate that it is the concentration of ions in SECL that should enter the IEM kinetic equations and not the total ion concentration as has been used so far. The concentration of ions in SECL estimated by using as a volume that of the average number of water molecules in the spherical shell is almost the same with that in the droplet core. (b) MICR (maximum ion concentration region) is the layer where there is an increased concentration of ions. There is an overlap between SECL and MICR, which is expected to increase with droplet radius.

Now we make the relation of MICR-SECL with the two-layer model in EMP. EPM focuses on SECL because the model uses the analogy with the structure of a double layer that appears next to a charged surface. For droplets with radius $<10 \mathrm{~nm}^{32}$ we think that the active area of the droplet should be extended by $\lambda_{\mathrm{PB}}$ in order to include MICR. If we consider a droplet of $10^{5} \mathrm{H}_{2} \mathrm{O}$ molecules, modeled by TIP3P at $T=350 \mathrm{~K}$ then the corresponding charge at the Rayleigh limit is $82.5 e$ and $R_{\mathrm{e}}=9.08 \mathrm{~nm}$, which yields $\lambda_{\mathrm{PB}}=1.29 \mathrm{~nm}$.

We have found that $\lambda_{\mathrm{PB}}$ increases from $1.0 \mathrm{~nm}$ to $1.35 \mathrm{~nm}$ at $T=350 \mathrm{~K}$ in droplets of $R_{\mathrm{e}}=$ $5.3 \mathrm{~nm}\left(2 \times 10^{5} \mathrm{H}_{2} \mathrm{O}\right.$ molecules $)$ to $R_{\mathrm{e}} \approx 10 \mathrm{~nm}$, respectively (data are shown in Table $\mathrm{S} 3$ in SI). In tiny droplets of approximately $10^{3} \mathrm{H}_{2} \mathrm{O}$ molecules MICR is extended to almost the entire droplet. It is straightforward to estimate the value of $\lambda_{\mathrm{PB}}$ at any temperature, solvent 
dielectric constant and droplet size. Thus, it is reasonable to extend SECL by approximately $1.0 \mathrm{~nm}$ in order to define a distinct droplet region that encompasses the highest ion concentration, carries the excess charge and it is subject to shape fluctuations. In droplets with radius $>10 \mathrm{~nm}$ the relative shape fluctuations are small thus, for this droplet size we expect MICR and SECL to overlap significantly.

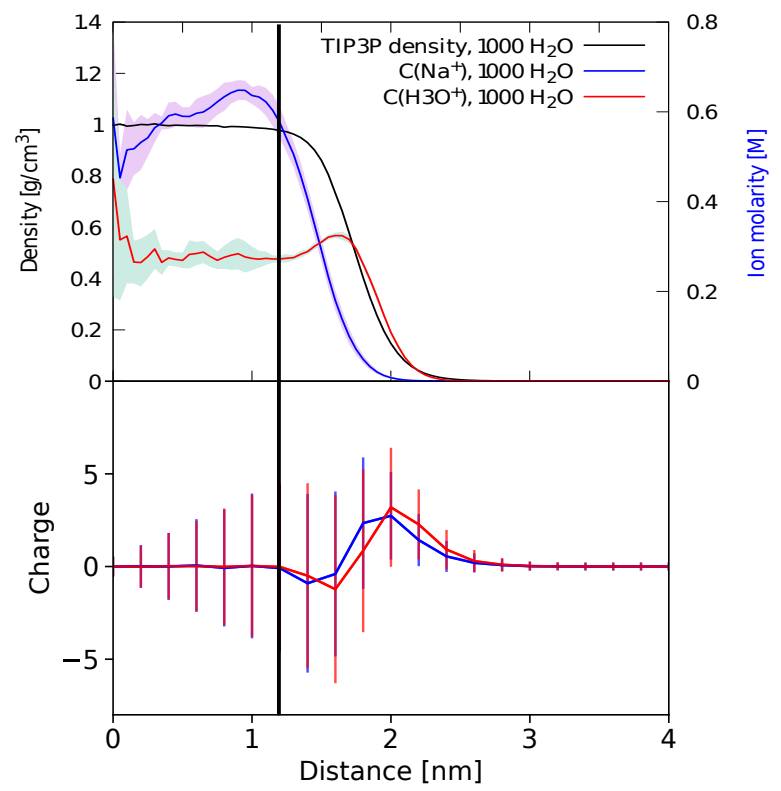

Figure 4: Droplet solvent density and hydronium ion radial distribution profile (upper panel). The $\mathrm{Na}^{+}$radial distribution profile is included for comparison. The drop comprises $\approx 880 \mathrm{H}_{2} \mathrm{O}$ molecules and $6 \mathrm{H}_{3} \mathrm{O}^{+}$ions at $T=350 \mathrm{~K}$. Total charge of the drop (lower panel). The black vertical line marks the onset of the surface excess charge layer. The blurry region shows the error bars. The production run is for $40 \mathrm{~ns}$. The standard deviation was calculated by using 5 blocks of raw data, each of $4^{4}$ frames.

The effect of the nature of the ions in their abundance in SECL is further examined in the cases of model hydronium ions and a protein complex. Figure 4 (a) shows the distribution of "hydronium" ions in a droplet of $\approx 1000 \mathrm{H}_{2} \mathrm{O}$ molecules and 6 ions at $T=350 \mathrm{~K}$. The hydronium distribution at $T=300 \mathrm{~K}$ is shown in
Fig. S8 in SI. The general features of the distribution are the same as that of the $\mathrm{Na}^{+}$ions but the hydronium reside much more in the outer water shells of the droplet than the $\mathrm{Na}^{+}$ions; $80 \%$ of hydronium ions reside in SECL vs. $55 \%$ of $\mathrm{Na}^{+}$ions. The thickness of the layer remains almost the same for the two types of ions. Even though the hydronium model omits important quantum effects it is demonstrated that it is possible for ions that carry features of the hydronium ion to have much higher concentration in SECL than $\mathrm{Na}^{+}$or $\mathrm{Cl}^{-}$ions.

Figure 5 (a) shows a typical snapshot of $1 \mathrm{JR}^{25+}$ in an aqueous droplet of $2 \times 10^{4} \mathrm{H}_{2} \mathrm{O}$ molecules and several co-ions and counterions (Table 1 ). In all the simulations $1 \mathrm{JR} 0^{25+}$ resides off the droplet COM. The charge distributions of aqueous droplets with various concentrations of simple ions shows that the surface excess charge layer has a thickness of $\approx 2.0 \mathrm{~nm}$ (the onset of the negative value of the charge density is at $4.6 \mathrm{~nm}$ and the end value is at $6.6 \mathrm{~nm}$ where the water density becomes $\approx 5 \times 10^{-4} \mathrm{~g} / \mathrm{cm}^{3}$ ). Therefore, in the presence of complex macromolecules SECL may be broader than that for simple ions.

\section{Structure of the surface excess charge layer}

Figure 6 (a) shows that in the presence of positive ions in aqueous drops, SECL undulates (negative trough followed by a positive peak) and Fig. 6 (b) that for negative ions there is a single negative trough. The difference in SECL in the presence of cations and anions is explained as follows: We consider the orientation of the $\mathrm{H}_{2} \mathrm{O}$ dipoles in pristine droplets as the reference structure. In a pristine droplet, there is an excess of hydrogen sites in the droplet periphery and thus, an excess of oxygen sites in the immediate (more in the interior) layer (Fig. 2 (b) and (c)) due to the presence of the interface. As a result the charge distribution shows undulation where a negative trough is followed by a positive peak. The cations enhance the presence of the hydrogen sites in the periphery and thus the undulation in SECL follows the same pattern as that of the pristine 

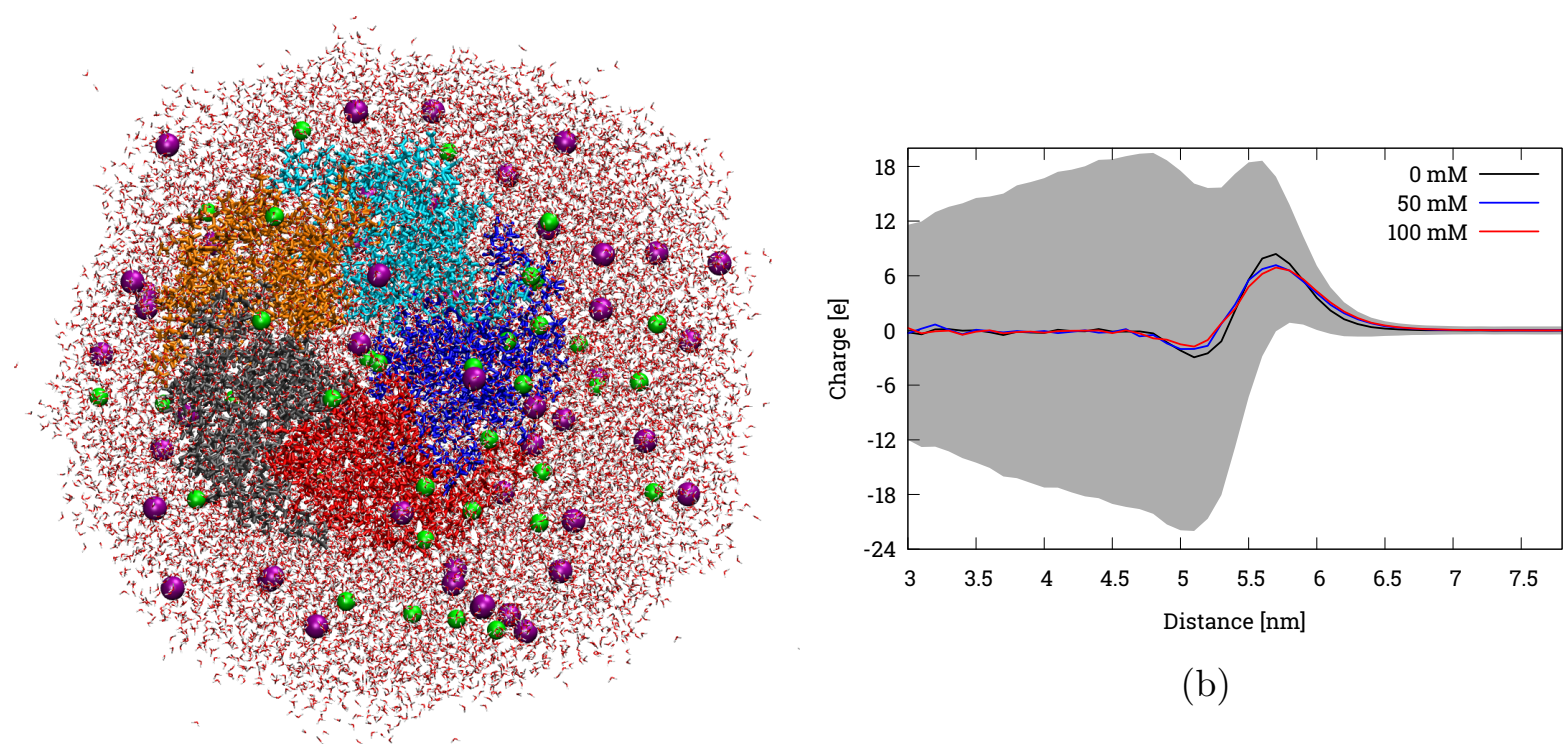

(b)

(a)

Figure 5: (a) Typical snapshot of an aqueous droplets comprised $1 \mathrm{JR}^{25+}, 14 \mathrm{Na}^{+}$ions and 36 pairs of $\mathrm{NaCl}$. The water molecules are depicted in red, the $\mathrm{Na}^{+}$ions in violet and the $\mathrm{Cl}^{-}$ions in green color. (b) Charge distribution measured from the droplet COM.

droplet. Differently, the presence of a single anion, as shown in Fig. 2 (c) has a significant effect in the lowering of the positive peak relative to that of a pristine aqueous droplet, while it does not affect the negative trough.

Figure 8 shows that the undulation in SECL approaches progressively a single trough as the number of negative ions increases. For one or two $\mathrm{Cl}^{-}$ions the undulation is maintained, but as the number of $\mathrm{Cl}^{-}$ions increases, the charge distribution leads to one trough. The lowering of the positive peak arises from the change in the orientation of the water molecules.

The solvent polarization charge accompanies the free charge. The orientation of the water dipoles is directly related to the polarization charge. For this reason we examine the distribution and average value of $\cos \phi$, where $\phi$ is the angle between the $\mathrm{H}_{2} \mathrm{O}$ dipole moment (directed from the oxygen site to the center of the line that connects the two hydrogen sites) and the vector that points from the droplet center of mass (COM) to the oxygen site of a water molecule. Figure 6 (c) and (d) show the average value $\langle\cos \phi\rangle$ as a function of distance from the droplet COM for droplets of different size and sign of the ion's charge. The corresponding distributions are shown in Fig. 7 (a) and (b) for a droplet of $880 \mathrm{H}_{2} \mathrm{O}$ molecules including $6 \mathrm{Na}^{+}$ions or $6 \mathrm{Cl}^{-}$ions, respectively. The distributions are estimated in spherical shells of thickness $0.5 \AA$ centered at the droplet COM. The same plots but for a droplet of $3 \times 10^{4}$ $\mathrm{H}_{2} \mathrm{O}$ molecules are shown in Fig. S9 in SI. The combination of Fig. 6 (c)-(d) and Fig. 7 (a)(b) show that for the smaller droplets of $\approx 880$ $\mathrm{H}_{2} \mathrm{O}$ molecules, there is a solvent polarization in the interior, while in the larger droplets of $\approx 3 \times 10^{4} \mathrm{H}_{2} \mathrm{O}$ molecules, the polarization - still present - is less pronounced. The interior polarization arises from the fact that it is likely to find ions in the interior (Fig. 3). The larger the droplet, the lower the concentration of the ions, which explains the less pronounced polarization in the larger droplets. The polarization charge for various droplet sizes with $\mathrm{Na}^{+}$ions is shown in Fig. S10 in SI. The maxima and minima of the polarization charge reduce as $1 / r$ where $r$ is the distance from the droplet COM. ${ }^{32}$ In SECL there are changes in the solvent polarization as indicated by the highlighted regions in Fig. 6 .

For droplets with $880 \mathrm{H}_{2} \mathrm{O}$ and $6 \mathrm{Na}^{+}$ions there are three characteristic distances from the droplet COM, which appear in the SECL (see Fig. 6 (a)). These are: $11.0 \pm 1.0 \AA$ where the surface excess charge starts to build-up, 


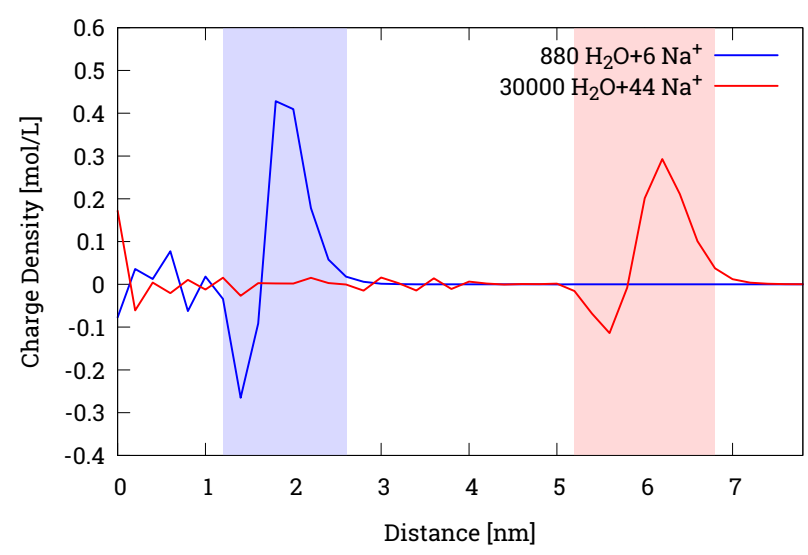

(a)

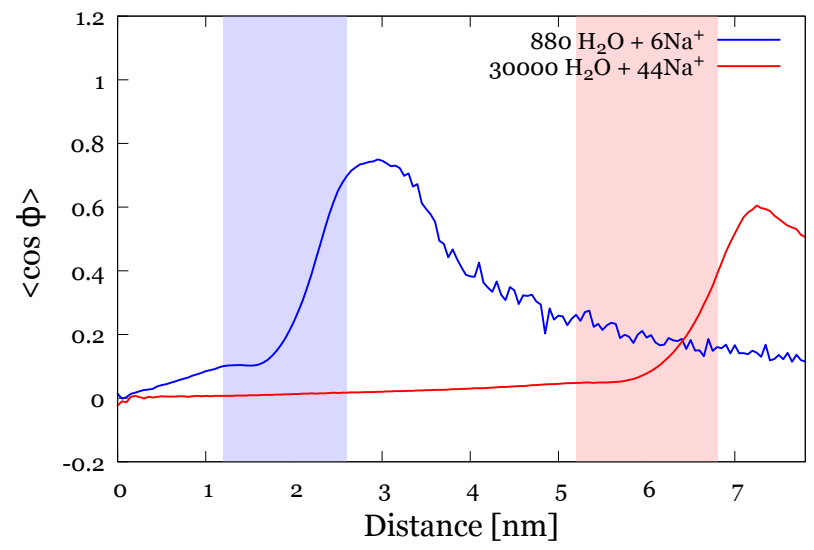

(c)

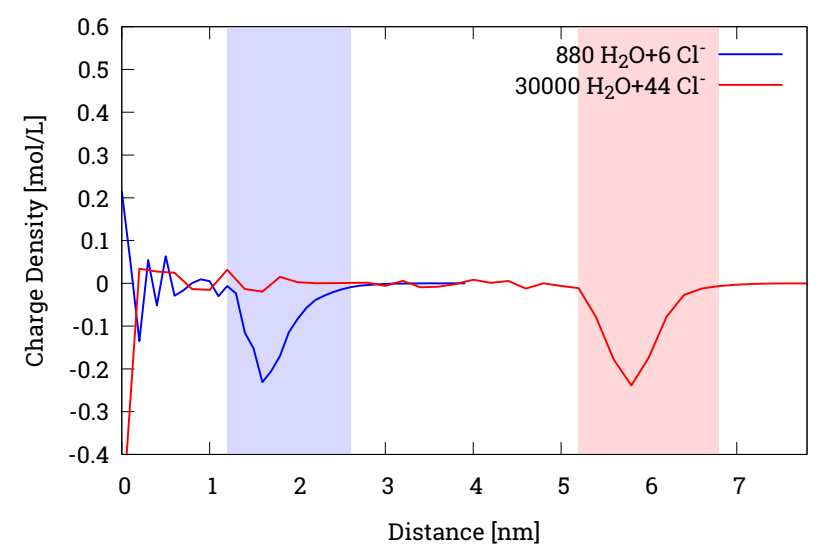

(b)

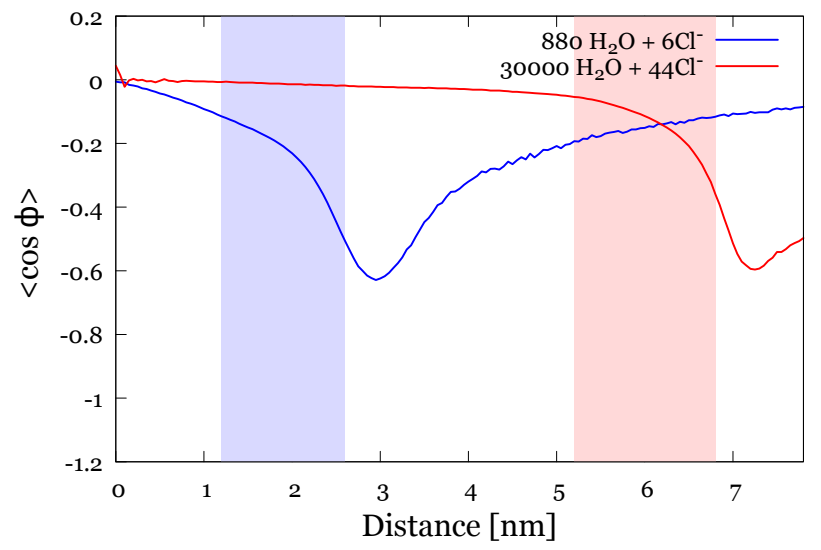

(d)

Figure 6: (a) Total charge density in droplets containing $\mathrm{Na}^{+}$; (b) as (a) but for $\mathrm{Cl}^{-}$ions. The shaded area denotes the surface excess charge layer. (c) $\langle\cos \phi\rangle$ as a function of the distance $(r)$ from the droplet COM in droplets containing $\mathrm{Na}^{+}$ions; (d) as (c) but for $\mathrm{Cl}^{-}$ions.

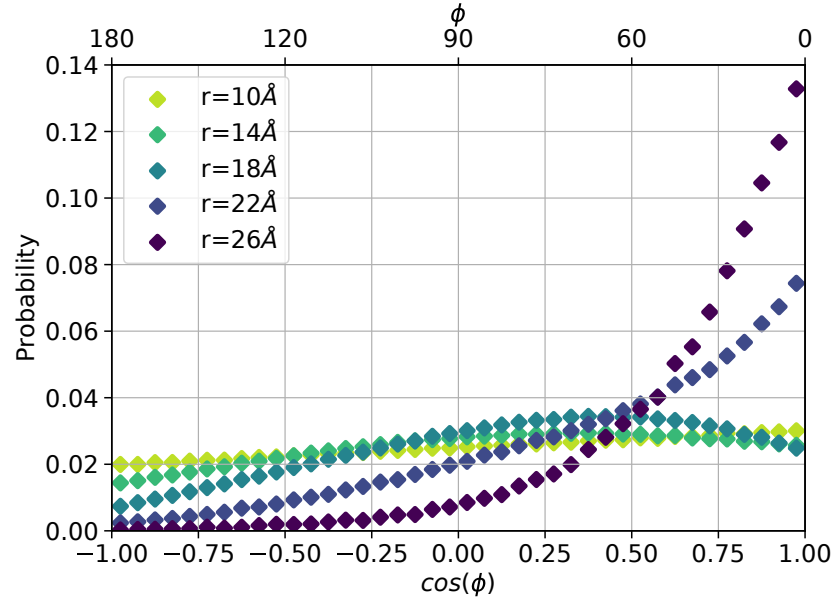

(a)

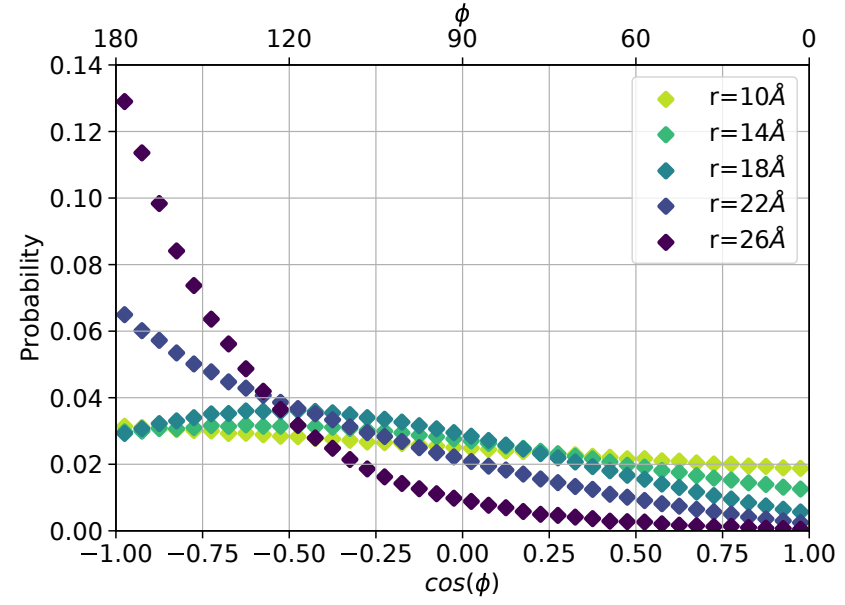

(b)

Figure 7: Distribution of $\cos (\phi)$ at selected distances from the droplet COM. The corresponding angles are shown on the top of the graphs. (a) $880 \mathrm{H}_{2} \mathrm{O}-6 \mathrm{Na}^{+}$ions and (b) $880 \mathrm{H}_{2} \mathrm{O}+6 \mathrm{Cl}^{-}$ions. 


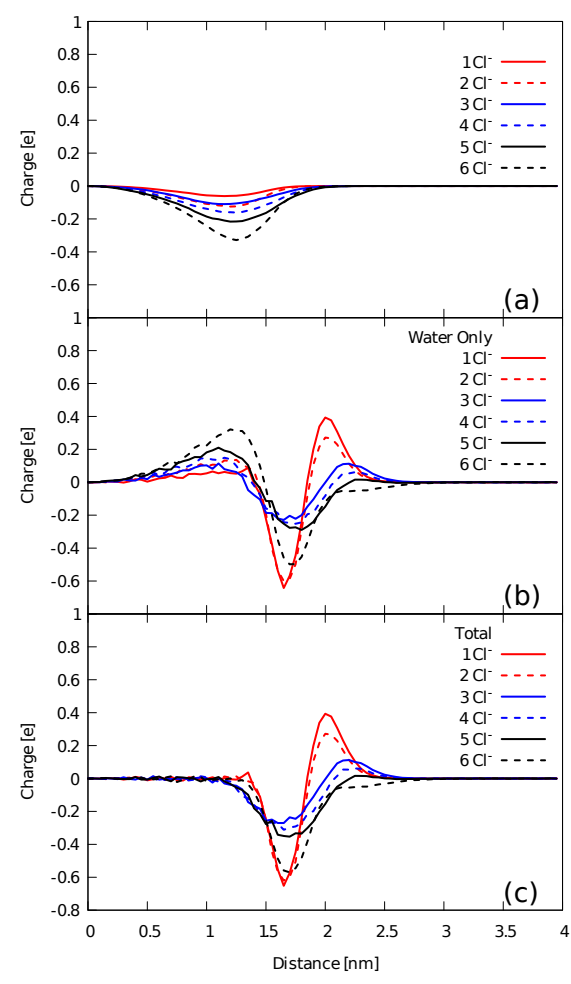

Figure 8: Charge distribution in droplets comprised $880 \mathrm{H}_{2} \mathrm{O}$ molecules and $1-6 \mathrm{Cl}^{-}$ions. (a) Ions only; (b) Water only; (c) Total (includes $\mathrm{Cl}^{-}, \mathrm{H}, \mathrm{O}$ sites).

at the top of the positive peak. Beyond the $19 \AA$, the density of the water is considerably reduced and all the charge is polarization charge. At these distances, we examine the orientation of the $\mathrm{H}_{2} \mathrm{O}$ dipole moment. All the graphs in Fig. 7 indicate that the angle distributions are quite broad, thus there is not a dominant angle. At $r=14 \AA$ the preferred $\cos \phi$ values are in $-0.25<\cos \phi<0.75\left(104.5^{\circ}<\phi<41.5^{\circ}\right)$ and at $r=19 \AA 0.0<\cos \phi<0.75\left(90^{\circ}<\right.$ $\left.\phi<41.5^{\circ}\right)$. At $r=19 \AA$ the probability of the negative $\cos \phi$ values have decreased considerably. In the presence of $\mathrm{Na}^{+}$ions, $\langle\cos \phi\rangle$ in the highlighted region (SECL) of Fig. 6 shows a small kink. A similar kink is not present in the droplets with $\mathrm{Cl}^{-}$ions. This difference in $\langle\cos \phi\rangle$ is associated with the different form of the surface charge distribution in the ions of different charge: a single negative trough for $\mathrm{Cl}^{-}$ions vs an undulated charge distribution for $\mathrm{Na}^{+}$ions. The angle variations are less pronounced for the larger droplets because of the lower ion concentration. In the very outer region of $r>19 \AA$, the angle distribution becomes sharper. At this distance the $\mathrm{H}_{2} \mathrm{O}$ are polarized by the entire droplet charge and not by a specific ion.

In the excess surface charge region, the number of hydrogen bonds reduces considerably relative to the droplet interior. The hydrogen bond distributions for $\mathrm{Na}^{+}$and $\mathrm{Cl}^{-}$ions are shown in Fig. S11 and Fig. S12 in SI, respectively. In droplets with diameter $\approx 4 \mathrm{~nm}$ the average number of hydrogen bonds per water molecule up to a distance $1.2 \mathrm{~nm}$ from the droplet COM is 2.9 while in the larger droplets up to $5.0 \mathrm{~nm}$ is 3.5. In SECL the number of hydrogen bonds in the smaller droplets reduces from 2.2 to 1.8 and in the larger droplet from 2.7 to 2.5 .

Now we examine the ion charge compensation by the solvent molecules. It is often assumed that the charge of the ions is compensated by the solvent because of its high dielectric constant. This is generally true, but there are cases that this assumption may not hold.

We computed the radial distribution function $(g(r))$ between the $\mathrm{Na}^{+}$ions as shown in Fig. 9. The $\mathrm{Na}^{+}-\mathrm{Na}^{+}$radial distribution function shows two significant peaks, at $\sim 3.7 \AA$ and $\sim 6.1 \AA$ that correspond to contact ion pairs (CIP) and solvent separated ion pair (SSIP), respectively. ${ }^{97-101}$ The relative distance of the ions has an interesting effect on the compensation of the ion charge by the solvent molecules. If we have a single $\mathrm{Na}^{+}$in an aqueous droplet, we find that the integral of the charge up to $8.0 \AA$ compensates the charge of the ion. When we have $6 \mathrm{Na}^{+}$ions in a droplet of $880 \mathrm{H}_{2} \mathrm{O}$ molecules, the charge of the single ion is not compensated by the $\mathrm{H}_{2} \mathrm{O}$ molecules found in a radius of $7.0 \AA$ around the ion. The charge compensation should also include the other ions. We attribute the lack of charge compensation by the solvent to the small droplet size. In the smaller droplets, two $\mathrm{Na}^{+}$ions are very likely to be found at a distance $<1.4 \mathrm{~nm}$, thus having their two immediate solvation shells interacting. The effect is less pronounced in larger droplet. 


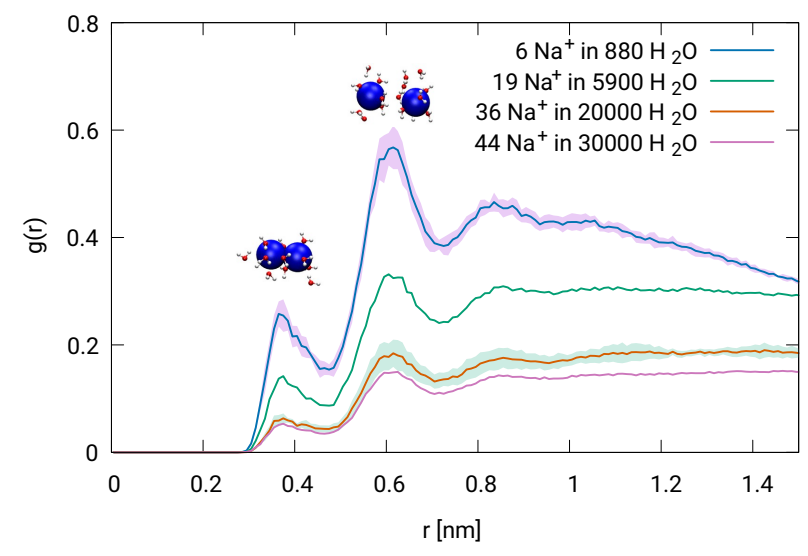

(a)

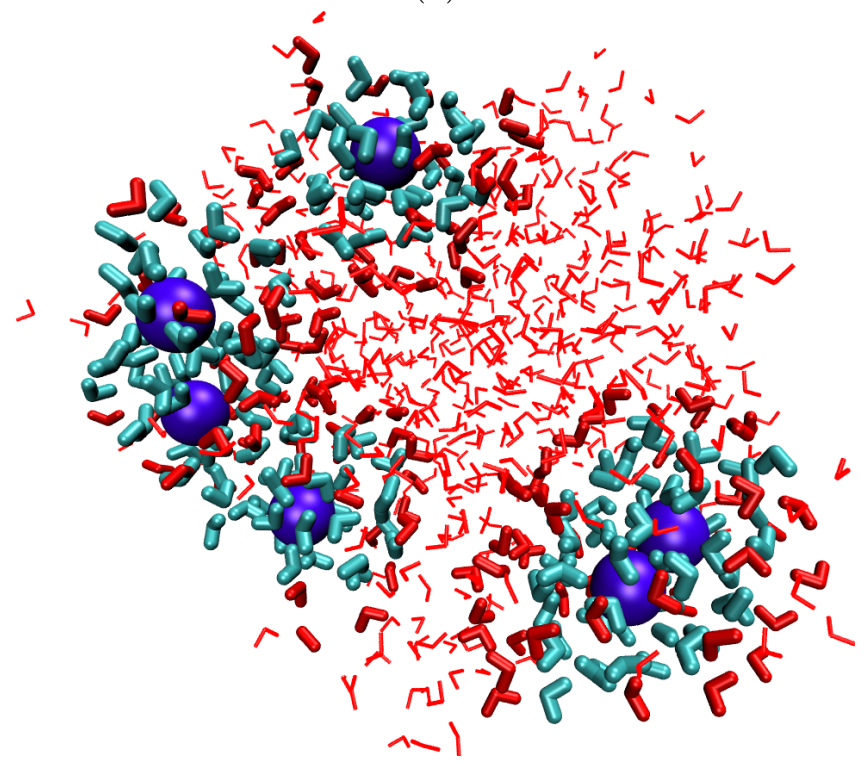

(b)

Figure 9: (a) Radial distribution function $(g(r))$ of $\mathrm{Na}^{+}$ions in aqueous droplets as a function of distance $r$ between any pair of ions. (b) Typical snapshot of a droplet of $880 \mathrm{H}_{2} \mathrm{O}$ molecules and $6 \mathrm{Na}^{+}$ions. Blue-colored $\mathrm{H}_{2} \mathrm{O}$ molecules are found at $r<6 \AA$ from a $\mathrm{Na}^{+}$center. The thick red-colored $\mathrm{H}_{2} \mathrm{O}$ molecules are at $8 \AA<r<$ $6 \AA$. The thin red-colored $\mathrm{H}_{2} \mathrm{O}$ molecules are the molecules that are not in the immediate vicinity of the ions. The snapshot shows in the encircled region the lack of sufficient solvation for charge compensation around the surface ions.

Charge compensation of the ions by the solvent may also not occur in ions that are very near the surface. In smaller droplets where the ion concentration is higher, the free charge that is near the surface may not be coordinated with sufficient number of water molecules to compensate its charge as shown in Fig. 9 (b). Our finding is also supported by the low dielectric constant of water detected under confinement. ${ }^{102,103}$

\section{Conclusions}

The surface excess charge of droplets has raised much discussion in the field of native mass spectrometry since the seminal works of Iribarne and Thomson ${ }^{14-16}$ and possibly earlier. Here for the first time we dissected the droplet structure and we identified two important layers: the surface excess charge layer (SECL) and the maximum ion concentration region (MICR).

Using molecular simulations we have found that in the presence of simple ions the surface charge is found in an outer layer of thickness $1.5 \mathrm{~nm}-1.7 \mathrm{~nm}$. The thickness is invariant not only with respect to droplet size but also with respect to the nature of the simple ions and the fine details of different force fields. In the presence of macroions the SECL may extend to $2.0 \mathrm{~nm}$. The proportion of ions (free charge) in SECL depends on the nature of the ion and the droplet size. For the same droplet size, $\mathrm{I}^{-}$and model hydronium ions show considerably higher concentration than the $\mathrm{Na}^{+}$and $\mathrm{Cl}^{-}$ions. In liquid droplets SECL is strongly affected by shape fluctuations, and it does not have the highest ion concentration. Using the decay length $\left(\lambda_{\mathrm{PB}}\right)$ of the ion distribution that arises from the non-linear Poisson-Boltzmann equation, we were able to identify the maximum ion concentration region (MICR). This region partially overlaps with SECL.

We showed that the structure of SECL changes with droplet size and sign of the ion's charge. The positive ions create an undulated charge distribution (like the one for pure water) while the negative ions at very low concentration exhibit an undulated charge distribution that progressively changes to a single trough when the ion concentration increases. The larger the droplet the lower the concentration of ions in SECL, thus the less pronounced is the effect of solvent polarization because of the presence of ions. In larger droplets the number of hydrogen bonds per molecule is main- 
tained high even in the surface excess charge region. The relative larger shape fluctuations in the smaller droplets and the larger surface to volume ratio reduce the number of hydrogen bonds in comparison to the larger droplets. The structure of SECL in smaller droplets indicate that emission of ions from smaller droplets is more facile relative to larger droplets.

We re-examine the relation of MICR-SECL with the bi-layer model in the equilibrium partition model (EPM) of C. Enke. EPM focuses on SECL because the model uses the analogy with the structure of a solution double layer that appears next to a charged surface. For droplets with radius $<10 \mathrm{~nm}^{32}$ we propose that the distinct region of the droplet may be extended by $\lambda_{\mathrm{PB}}$ in order to include MICR. We have found that $\lambda_{\mathrm{PB}}$ increases from $1.0 \mathrm{~nm}$ to $1.35 \mathrm{~nm}$ at $T=350 \mathrm{~K}$ for a droplet radius from $5.3 \mathrm{~nm}\left(2 \times 10^{5} \mathrm{H}_{2} \mathrm{O}\right.$ molecules $)$ to $\approx 10 \mathrm{~nm}$, respectively. In tiny droplets of approximately $10^{3} \mathrm{H}_{2} \mathrm{O}$ molecules MICR is extended to almost the entire droplet. The combination of MICR and SECL may create a droplet layer of approximate thickness of $3.0 \mathrm{~nm}$ that is characterized by highest ion concentration, it is subject to shape fluctuations and especially in the smaller droplets the solvent polarization leads to large variations in the number of hydrogen bonds from the droplet interior to its surface. In a previous article, ${ }^{31}$ we have found the radial distribution of a charged polyhistidine of various charge states in an charged aqueous droplet. Comparison of the radial distribution of a polyhistidine with the combined SECL-MICR width reported here suggests that proteins may access MICR, at least in droplets with dimensions of several nanometers in diameter. Consequently part of a protein, or of a macromolecule in the more general case, can be exposed to a higher concentration of free ions and differently oriented solvent, which may affect its charge state.

IEM and the EPM give emphasis on different aspects of the droplet chemistry that may explain the abundances in the mass spectra. The former focuses on the kinetics of ion evaporation and the latter on the equilibrium structure of the droplet. At a first glance IEM and EPM may appear disjoint mechanisms. However, it is possible to combine them, by using the concentration of ions in SECL as input in the kinetic equation of IEM. We remind that the model in IEM of Iribarne and Thomson considers the total number of ions in the droplet. Fluctuations may create the electric field for ion emission and may lead to ion release much below the Rayleigh limit. The smaller the droplet, the larger the relative shape fluctuations, the more likely the ion to escape much before the Rayleigh limit. The inclusion of shape fluctuations and thus, the fluctuations in the electric field would provide missing components in IEM. In atmospheric aerosols where the droplets are charged much below the Rayleigh limit, the IEM will be the mechanism to be followed for droplet disintegration.

In continuum models for electrosprayed droplet it has been often assumed that the ionic charge is compensated by the solvent. ${ }^{16}$ We have found that this may not be the case in smaller droplets with diameter $<4 \mathrm{~nm}$ and high concentration of ions. In this case the ion charge is not compensated only by the solvent polarization charge but by the total charge that includes both solvent and other ions. Thus, there are correlations in the locations of the ions and for this reason we cannot assume that the energy of the single ion is not affected by the presence of the others as it has been assumed in variations of the IEM model. ${ }^{16}$ In droplets with diameter $>4 \mathrm{~nm}$ the assumption of the energy independence of the single ion should be also tested because of high ion concentration due to counterions and multiply charged macroions.

In terms of computational methodology, we presented an extensive discussion in the "Models and Simulation Methods" section that supports that the preferred simulation set-up for the computation of droplet equilibrium properties is the enclosure of the droplet in a spherical cavity where it is in equilibrium with its vapor. The canonical ensemble is preferred to the microcanonical because known weaknesses of the microcanonical molecular dynamics prevent the performance of long trajectories, which are necessary to generate converging ion distributions. Constant energy MD runs should be used in the efficient study of fragmentation dy- 
namics of charged droplets in combination with free energy surfaces along collective reaction coordinates. $^{86}$

The next step in this study is to examine the concentration of a mixture of ions in the surface excess charge layer. The abundance of the ions in the surface excess charge region may change as a function of their overall percentage ratio in the droplet. Moreover, the emission of ions will change the droplet composition, thus there may be a redistribution of ions in the outer shell. It is also to be examined in future work whether the preference for the release of certain types of ions is determined by the composition of the surface excess charge layer in combination with the shape fluctuations. The effect of the nature of the solvent in the ion distribution and the role of the quadrupole moment in the polarization charge are also questions to be investigated.

\section{Supplementary Material}

See supplementary material of (a) force field parameters, (b) radial charge distribution profiles, (c) dipole angle distribution profiles and (d) Hydrogen bond profiles.

Acknowledgement S.C. thanks Prof. D. Frenkel, Department of Chemistry, University of Cambridge, UK, Prof. S. S. Xantheas, Pacific Northwest National Laboratory, and Dr. Anatoly Malevanets, The University of Western Ontario for discussions on the stability of charged systems. S.C. also thanks Professor Vicki Wysocki, Department of Chemistry, The Ohio State University for suggesting to study the cholera toxin protein assembly. Professor Christie G. Enke and Professor R. Zare, Stanford University are thanked for discussions on the droplet surface excess charge layer. S.C. acknowledges an NSERC-Discovery grant (Canada) for funding this research. V.K. acknowledges the province of Ontario and the University of Western Ontario for the Queen Elizabeth II Graduate Scholarship in Science and Technology. SHARCNET and Compute Canada are acknowledged for providing the computing facilities.

\section{References}

(1) Kebarle, P.; Verkerk, U. H. Electrospray: from ions in solution to ions in the gas phase, what we know now. Mass Spectrom. Rev. 2009, 28, 898-917.

(2) Wilm, M. Principles of electrospray ionization. Mol. Cell. Proteomics 2011, 10, M111-009407.

(3) Loo, R. R. O.; Lakshmanan, R.; Loo, J. A. What protein charging (and supercharging) reveal about the mechanism of electrospray ionization. J. Am. Soc. Mass Spectrom. 2014, 25, 16751693.

(4) Mehmood, S.; Allison, T. M.; Robinson, C. V. Mass spectrometry of protein complexes: From origins to applications. Annu. Rev. Phys. Chem. 2015, 66, 453474.

(5) Hirabayashi, A.; Sakairi, M.; Koizumi, H. Sonic spray ionization method for atmospheric pressure ionization mass spectrometry. Anal. Chem. 1994, 66, 45574559 .

(6) Blakley, C.; Vestal, M. Thermospray interface for liquid chromatography/mass spectrometry. Anal. Chem. 1983, 55, 750-754.

(7) Monge, M. E.; Harris, G. A.; Dwivedi, P.; Fernandez, F. M. Mass spectrometry: recent advances in direct open air surface sampling/ionization. Chem. Rev. 2013, 113, 2269-2308.

(8) Teunissen, S. F.; Eberlin, M. N. Transferring Ions from Solution to the Gas Phase: The Two Basic Principles. J. Am. Soc. Mass Spectrom. 2017, 28, 2255-2261.

(9) Sakairi, M.; Kambara, H. Atmospheric pressure spray ionization for liquid chromatography/mass spectrometry. Anal. Chem. 1989, 61, 1159-1164. 
(10) Bruins, A. P.; Covey, T. R.; Henion, J. D. Ion spray interface for combined liquid chromatography/atmospheric pressure ionization mass spectrometry. Anal. Chem. 1987, 59, 2642-2646.

(11) Kambara, H. Sample introduction system for atmospheric pressure ionization mass spectrometry of nonvolatile compounds. Anal. Chem. 1982, 54, 143-146.

(12) Devereaux, Z. J.; Reynolds, C. A.; Fischer, J. L.; Foley, C. D.; DeLeeuw, J. L.; Wager-Miller, J.; Narayan, S. B.; Mackie, K.; Trimpin, S. Matrix-Assisted Ionization on a Portable Mass Spectrometer: Analysis Directly from Biological and Synthetic Materials. Anal. Chem. 2016, 88, 10831-10836.

(13) Peacock, P. M.; Zhang, W.-J.; Trimpin, S. Advances in ionization for mass spectrometry. Anal. Chem. 2016, 89, 372-388.

(14) Iribarne, J. V.; Thomson, B. A. On the evaporation of small ions from charged droplets. J. Chem. Phys. 1976, 64, 22872294.

(15) Thomson, B.; Iribarne, J. Field induced ion evaporation from liquid surfaces at atmospheric pressure. J. Chem. Phys. 1979, 71, 4451-4463.

(16) Labowsky, M.; Fenn, J.; de la Mora, J. F. A continuum model for ion evaporation from a drop: effect of curvature and charge on ion solvation energy. Anal. Chim. 2000, 406, 105-118.

(17) Fenn, J. B.; Rosell, J.; Meng, C. K. In electrospray ionization, how much pull does an ion need to escape its droplet prison? J. Am. Soc. Mass. Spectrom. 1997, 8, 1147-1157.

(18) Fenn, J. B. Ion formation from charged droplets: roles of geometry, energy, and time. J. Am. Soc. Mass. Spectrom. 1993, $4,524-535$.
(19) de la Mora, J. F. Electrospray ionization of large multiply charged species proceeds via Dole's charged residue mechanism. Anal. Chim. Acta 2000, 406, 93104.

(20) Dole, M.; Mack, L.; Hines, R.; Mobley, R.; Ferguson, L.; Alice, M. d. Molecular beams of macroions. J. Chem. Phys. 1968, 49, 2240-2249.

(21) Labowsky, M. Discrete charge distributions in dielectric droplets. J. Colloid Interface Sci. 1998, 206, 19-28.

(22) Enke, C. G. A predictive model for matrix and analyte effects in electrospray ionization of singly-charged ionic analytes. Anal. Chem. 1997, 69, 4885-4893.

(23) Constantopoulos, T. L.; Jackson, G. S.; Enke, C. G. Challenges in achieving a fundamental model for ESI. Anal. Chim. Acta 2000, 406, 37-52.

(24) Hogan Jr, C. J.; Carroll, J. A.; Rohrs, H. W.; Biswas, P.; Gross, M. L. Combined charged residue-field emission model of macromolecular electrospray ionization. Anal. Chem. 2009, 81, 369377.

(25) Chen, X.; Cooks, R. G. Accelerated reactions in field desorption mass spectrometry. J. Mass Spectrom. 2018, 53, 942946.

(26) Schrader, R. L.; Fedick, P. W.; Mehari, T. F.; Cooks, R. G. Accelerated Chemical Synthesis: Three Ways of Performing the Katritzky Transamination Reaction. J. Chem. Educ. 2019, 96, 360-365.

(27) Lee, J. K.; Banerjee, S.; Nam, H. G.; Zare, R. N. Acceleration of reaction in charged microdroplets. Q. Rev. Biophys. 2015, 48, 437-444.

(28) Ingram, A. J.; Boeser, C. L.; Zare, R. N. Going beyond electrospray: mass spectrometric studies of chemical reactions in 
and on liquids. Chem. Sci. 2016, 7, 3955.

(29) Sahraeian, T.; Kulyk, D. S.; BaduTawiah, A. K. Droplet Imbibition Enables Non-Equilibrium Interfacial Reactions in Charged Microdroplets. Langmuir 2019, 35, 14451-14457.

(30) Kulyk, D. S.; Miller, C. F.; BaduTawiah, A. K. Reactive charged droplets for reduction of matrix effects in electrospray ionization mass spectrometry. Anal. Chem. 2015, 87, 10988-10994.

(31) Kwan, V.; Malevanets, A.; Consta, S. Where do the ions reside in a highly charged droplet? J. Phys. Chem. A 2019, 123, 9298-9310, PMID: 31589448.

(32) Kwan, V.; Consta, S. Bridging electrostatic properties between nanoscopic and microscopic highly charged droplets. Chem. Phys. Lett. 2020, 746, 137238.

(33) Consta, S.; Kapral, R. Dynamics of proton transfer in mesoscopic clusters. J. Chem. Phys. 1996, 104, 4581-4590.

(34) Constantopoulos, T. L.; Jackson, G. S.; Enke, C. G. Effects of salt concentration on analyte response using electrospray ionization mass spectrometry. $J$. Am. Soc. Mass Spectrom. 1999, 10, 625634.

(35) Rayleigh, L. XX. On the equilibrium of liquid conducting masses charged with electricity. Philos. Mag. 1882, 14, 184186.

(36) Hendricks, C.; Schneider, J. Stability of a conducting droplet under the influence of surface tension and electrostatic forces. Am. J. Phys 1963, 31, 450-453.

(37) Peters, J. Rayleigh's electrified water drops. Eur. J. Phys. 1980, 1, 143.

(38) Consta, S.; Malevanets, A. Disintegration mechanisms of charged nanodroplets: novel systems for applying methods of activated processes. Mol. Simul. 2015, 41, 73-85.

(39) Oh, M. I.; Malevanets, A.; Paliy, M.; Frenkel, D.; Consta, S. When droplets become stars: charged dielectric droplets beyond the Rayleigh limit. Soft Matter 2017, 13, 8781-8795.

(40) Grimm, R. L.; Beauchamp, J. Evaporation and discharge dynamics of highly charged multicomponent droplets generated by electrospray ionization. J. Phys. Chem. A 2009, 114, 1411-1419.

(41) Pickens, J. C.; Merritt, E. A.; Ahn, M.; Verlinde, C. L.; Hol, W. G.; Fan, E. Anchor-Based Design of Improved Cholera Toxin and E. coli Heat-Labile Enterotoxin Receptor Binding Antagonists that Display Multiple Binding Modes. Chemistry \& Biology 2002, 9, 215-224.

(42) Phillips, J. C.; Braun, R.; Wang, W.; Gumbart, J.; Tajkhorshid, E.; Villa, E.; Chipot, C.; Skeel, R. D.; Kalé, L.; Schulten, K. Scalable molecular dynamics with NAMD. J. Comput. Chem. 2005, 26, 1781-1802.

(43) Humphrey, W.; Dalke, A.; Schulten, K. VMD: Visual Molecular Dynamics. J. Mol. Graphics 1996, 14, 33-38.

(44) Jorgensen, W. L.; Jenson, C. Temperature dependence of TIP3P, SPC, and TIP4P water from NPT Monte Carlo simulations: Seeking temperatures of maximum density. J. Comput. Chem. 1998, 19, 1179-1186.

(45) Abascal, J. L. F.; Vega, C. A general purpose model for the condensed phases of water: TIP4P/2005. J. Chem. Phys. 2005, 123, 234505.

(46) Noskov, S. Y.; Roux, B. Control of Ion Selectivity in LeuT: Two Na+ Binding Sites with Two Different Mechanisms. J. Mol. Biol. 2008, 377, $804-818$. 
(47) Beglov, D.; Roux, B. Finite representation of an infinite bulk system: Solvent boundary potential for computer simulations. J. Chem. Phys. 1994, 100, 90509063.

(48) Chandrasekhar, J.; Spellmeyer, D. C.; Jorgensen, W. L. Energy component analysis for dilute aqueous solutions of lithium(1+), sodium(1+), fluoride(1-), and chloride(1-) ions. J. Am. Chem. Soc. 1984, 106, 903-910.

(49) McDonald, N. A.; Duffy, E. M.; Jorgensen, W. L. Monte Carlo investigations of selective anion complexation by a bis(phenylurea) p-tertbutylcalix[4]arene. J. Am. Chem. Soc. 1998, 120, 5104-5111.

(50) Eigen, M. Proton transfer, acid-base catalysis, and enzymatic hydrolysis. Part I: elementary processes. Angew. Chem. Int. Ed. 1964, 3, 1-19.

(51) Schuster, P.; Zundel, G.; Sandorfy, C. Hydrogen bond; recent developments in theory and experiments; North-Holland Publishing Company, 1976.

(52) Asmis, K. R.; Pivonka, N. L.; Santambrogio, G.; Brümmer, M.; Kaposta, C.; Neumark, D. M.; Wöste, L. Gas-phase infrared spectrum of the protonated water dimer. Science 2003, 299, 1375-1377.

(53) Vendrell, O.; Gatti, F.; Meyer, H.-D. Dynamics and infrared spectroscopy of the protonated water dimer. Angew. Chem. Int. Ed. 2007, 46, 6918-6921.

(54) Valeev, E. F.; Schaefer III, H. F. The protonated water dimer: Brueckner methods remove the spurious C 1 symmetry minimum. J. Chem. Phys. 1998, 108, 7197-7201.

(55) Vendrell, O.; Gatti, F.; Meyer, H.-D. Full dimensional (15-dimensional) quantumdynamical simulation of the protonated water dimer. II. Infrared spectrum and vibrational dynamics. J. Chem. Phys. 2007, 12\%, 184303.

(56) Pieniazek, P. A.; VandeVondele, J.; Jungwirth, P.; Krylov, A. I.; Bradforth, S. E. Electronic structure of the water dimer cation. J. Phys. Chem. A 2008, 112, 6159-6170.

(57) Spura, T.; Elgabarty, H.; Kühne, T. D. "On-the-fly" coupled cluster pathintegral molecular dynamics: impact of nuclear quantum effects on the protonated water dimer. Phys. Chem. Chem. Phys. 2015, 17, 14355-14359.

(58) Auer, A. A.; Helgaker, T.; Klopper, W. Accurate molecular geometries of the protonated water dimer. Phys. Chem. Chem. Phys. 2000, 2, 2235-2238.

(59) Wales, D. J. Rearrangements and tunneling splittings of protonated water dimer. J. Chem. Phys. 1999, 110, 10403-10409.

(60) Chang, H.-C.; Wu, C.-C.; Kuo, J.-L. Recent advances in understanding the structures of medium-sized protonated water clusters. Int. Rev. Phys. Chem. 2005, 24, 553-578.

(61) Tachikawa, M.; Shiga, M. Geometrical $\mathrm{H} / \mathrm{D}$ isotope effect on hydrogen bonds in charged water clusters. J. Am. Chem. Soc. 2005, 127, 11908-11909.

(62) Park, M.; Shin, I.; Singh, N. J.; Kim, K. S. Eigen and Zundel forms of small protonated water clusters: structures and infrared spectra. J. Phys. Chem. A 2007, 111, 10692-10702.

(63) Wang, H.; Agmon, N. Reinvestigation of the infrared spectrum of the gasphase protonated water tetramer. $J$. Phys. Chem. A 2017, 121, 3056-3070.

(64) Mouhat, F.; Sorella, S.; Vuilleumier, R.; Saitta, A. M.; Casula, M. Fully quantum description of the Zundel ion: combining variational quantum Monte Carlo with path integral Langevin dynamics. $J$. 
Chem. Theory Comput. 2017, 13, 24002417.

(65) Xantheas, S. S.; Dunning Jr, T. H. Ab initio studies of cyclic water clusters $(\mathrm{H} 2 \mathrm{O}) \mathrm{n}, \mathrm{n}=1-6$. I. Optimal structures and vibrational spectra. J. Chem. Phys. 1993, 99, 8774-8792.

(66) Yu, Q.; Bowman, J. M. High-level quantum calculations of the IR spectra of the Eigen, Zundel, and ring isomers of $\mathrm{H}+$ (H2O) 4 find a single match to experiment. J. Am. Chem. Soc. 2017, 139, 10984-10987.

(67) Yu, Q.; Bowman, J. M. Classical, Thermostatted Ring Polymer, and Quantum VSCF/VCI Calculations of IR Spectra of H7O3+ and H9O4+ (Eigen) and Comparison With Experiment. J. Phys. Chem. A 2019,

(68) Burnham, C. J.; Petersen, M. K.; Day, T. J.; Iyengar, S. S.; Voth, G. A. The properties of ion-water clusters. II. Solvation structures of $\mathrm{Na}+, \mathrm{Cl}-$, and $\mathrm{H}+$ clusters as a function of temperature. $J$. Chem. Phys. 2006, 124, 024327.

(69) Petersen, M. K.; Iyengar, S. S.; Day, T. J.; Voth, G. A. The hydrated proton at the water liquid/vapor interface. J. Phys. Chem. B 2004, 108, 14804-14806.

(70) Hvelplund, P.; Kurten, T.; Støchkel, K.; Ryding, M. J.; Nielsen, S. B.; Uggerud, E. Stability and structure of protonated clusters of ammonia and water, $\mathrm{H}+(\mathrm{NH} 3) \mathrm{m}(\mathrm{H} 2 \mathrm{O})$ n. J. Phys. Chem. A 2010, 114, 7301-7310.

(71) Silverstein, T. P. The Aqueous Proton Is Hydrated by More Than One Water Molecule: Is the Hydronium Ion a Useful Conceit? J. Chem. Educ. 2014, 91, 608-610.

(72) Colussi, A. J. Can the $\mathrm{pH}$ at the air/water interface be different from the
pH of bulk water? Proc. Natl. Acad. Sci. USA 2018, 115, E7887.

(73) Chuev, G. N.; Fedorov, M. V.; Chiodo, S.; Russo, N.; Sicilia, E. Hydration of ionic species studied by the reference interaction site model with a repulsive bridge correction. J. Comp. Chem. 2008, 29, 2406-2415.

(74) Berman, H. M.; Westbrook, J.; Feng, Z.; Gilliland, G.; Bhat, T. N.; Weissig, H.; Shindyalov, I. N.; Bourne, P. E. The protein data bank. Nucleic Acids Res. 2000, 28, 235-242.

(75) http://www.rcsb.org.

(76) Vega, C.; de Miguel, E. Surface tension of the most popular models of water by using the test-area simulation method. J. Chem. Phys. 2007, 126, 154707.

(77) Zakharov, V. V.; Brodskaya, E. N.; Laaksonen, A. Surface tension of water droplets: A molecular dynamics study of model and size dependencies. J. Chem. Phys. 1997, 10\%, 10675-10683.

(78) Soleilhac, A.; Dagany, X.; Dugourd, P.; Girod, M.; Antoine, R. Correlating droplet size with temperature changes in electrospray source by optical methods. Anal. Chem. 2015, 87, 8210-8217.

(79) Gibson, S. C.; Feigerle, C. S.; Cook, K. D. Fluorometric measurement and modeling of droplet temperature changes in an electrospray plume. Anal. Chem. 2013, $86,464-472$.

(80) Consta, S.; Oh, M. I.; Kwan, V.; Malevanets, A. Strengths and Weaknesses of Molecular Simulations of Electrosprayed Droplets. J. Am. Soc. Mass Spectrom. 2018, 29, 2287-2296.

(81) Bixon, M.; Jortner, J. Energetic and thermodynamic size effects in molecular clusters. J. Chem. Phys. 1989, 91, 16311642 . 
(82) Carignano, M. Monte Carlo simulations of small water clusters: microcanonical vs canonical ensemble. Chemical physics letters 2002, 361, 291-297.

(83) Ray, J. R.; Graben, H. Small systems have non-Maxwellian momentum distributions in the microcanonical ensemble. Physical Review A 1991, 44, 6905.

(84) Michaelian, K.; Santamaría-Holek, I. Dynamics and Thermodynamics of Nanoclusters. Entropy 2015, 17, 7133-7148.

(85) Allen, M.; Tildesley, D. Computer Simulation of Liquids; Oxford: Clarendon Pr, 1987.

(86) Consta, S.; Mainer, K. R.; Novak, W. Fragmentation mechanisms of aqueous clusters charged with ions. J. Chem. Phys. 2003, 119, 10125-10132.

(87) Roux, B.; Yu, H. A.; Karplus, M. Molecular basis for the Born model of ion solvation. J. Phys. Chem. 1990, 94, 46834688.

(88) Rashin, A. A.; Honig, B. Reevaluation of the Born model of ion hydration. J. Phys. Chem. 1985, 89, 5588-5593.

(89) Jayaram, B.; Fine, R.; Sharp, K.; Honig, B. Free energy calculations of ion hydration: an analysis of the Born model in terms of microscopic simulations. $J$. Phys. Chem. 1989, 93, 4320-4327.

(90) Hirata, F.; Redfern, P.; Levy, R. M. Viewing the Born model for ion hydration through a microscope. Int. J. Quantum Chem. 1988, 34, 179-190.

(91) Bucher, M.; Porter, T. L. Analysis of the Born model for hydration of ions. $J$. Phys. Chem. 1986, 90, 3406-3411.

(92) Ashbaugh, H. S. Convergence of molecular and macroscopic continuum descriptions of ion hydration. J. Phys. Chem. B 2000, 104, 7235-7238.
(93) Rajamani, S.; Ghosh, T.; Garde, S. Size dependent ion hydration, its asymmetry, and convergence to macroscopic behavior. J. Chem. Phys. 2004, 120, 44574466 .

(94) Hummer, G.; Pratt, L. R.; Garcia, A. E. Ion sizes and finite-size corrections for ionic-solvation free energies. J. Chem. Phys. 1997, 107, 9275-9277.

(95) Grossfield, A. Dependence of ion hydration on the sign of the ion's charge. J. Chem. Phys. 2005, 122, 024506.

(96) Malevanets, A.; Consta, S. Variation of droplet acidity during evaporation. $J$. Chem. Phys. 2013, 138, 184312.

(97) Ciccotti, G.; Ferrario, M.; Hynes, J. T.; Kapral, R. Dynamics of ion pair interconversion in a polar solvent. J. Chem. Phys. 1990, 93, 7137-7147.

(98) Dang, L. X.; Pettitt, B. M.; Rossky, P. J. On the correlation between like ion pairs in water. J. Chem. Phys. 1992, 96, 40464047.

(99) Pettitt, B. M.; Rossky, P. J. Alkali halides in water: Ion-solvent correlations and ion-ion potentials of mean force at infinite dilution. J. Chem. Phys. 1986, $84,5836-5844$.

(100) Consta, S.; Kapral, R. Ionization reactions of ion complexes in mesoscopic water clusters. J. Chem. Phys. 1999, 111, 10183-10191.

(101) Laria, D.; Fernández-Prini, R. Molecular dynamics study of water clusters containing ion pairs: From contact to dissociation. J. Chem. Phys. 1995, 102, 76647673 .

(102) Fumagalli, L.; Esfandiar, A.; Fabregas, R.; Hu, S.; Ares, P.; Janardanan, A.; Yang, Q.; Radha, B.; Taniguchi, T.; Watanabe, K. et al. Anomalously low dielectric constant of confined water. Science 2018, 360, 1339-1342. 
(103) Schlaich, A.; Knapp, E. W.; Netz, R. R. Water dielectric effects in planar confinement. Phys. Rev. Lett. 2016, 11\%, 048001. 